Chapman University

Chapman University Digital Commons

Food Science Faculty Articles and Research

Science and Technology Faculty Articles and

Research

$3-12-2020$

\title{
Detection of Fish Fillet Substitution and Mislabeling Using Multimode Hyperspectral Imaging Techniques
}

Jianwei Qin

USDA/ARS Environmental Microbial and Food Safety Laboratory

Fartash Vasefı

SafetySpect Inc.

Rosalee S. Hellberg

Chapman University, hellberg@chapman.edu

Alireza Akhbardeh

SafetySpect Inc.

Rachel B. Isaacs

Chapman University

See next page for additional authors

Follow this and additional works at: https://digitalcommons.chapman.edu/food_science_articles

Part of the Aquaculture and Fisheries Commons, Food Biotechnology Commons, Food Microbiology Commons, Food Processing Commons, and the Other Food Science Commons

\section{Recommended Citation}

Qin, J., Vasefi, F., Hellberg, R.S., Akhbardeh, A., Isaacs, R.B., Yilmaz, A.G., Hwang, C., Baek, I., Schmidt, W.F., Kim, M.S. (2020). Detection of fish fillet substitution and mislabeling using multimode hyperspectral imaging techniques. Food Control, 114, 107234. https://doi.org/10.1016/j.foodcont.2020.107234

This Article is brought to you for free and open access by the Science and Technology Faculty Articles and Research at Chapman University Digital Commons. It has been accepted for inclusion in Food Science Faculty Articles and Research by an authorized administrator of Chapman University Digital Commons. For more information, please contact laughtin@chapman.edu. 


\section{Detection of Fish Fillet Substitution and Mislabeling Using Multimode Hyperspectral Imaging Techniques}

\section{Comments}

NOTICE: this is the author's version of a work that was accepted for publication in Food Control. Changes resulting from the publishing process, such as peer review, editing, corrections, structural formatting, and other quality control mechanisms may not be reflected in this document. Changes may have been made to this work since it was submitted for publication. A definitive version was subsequently published in Food Control, volume 114, in 2020. https://doi.org/10.1016/j.foodcont.2020.107234

The Creative Commons license below applies only to this version of the article.

\section{Creative Commons License}

\section{(c) (i) (3)}

This work is licensed under a Creative Commons Attribution-Noncommercial-No Derivative Works 4.0 License.

\section{Copyright}

Elsevier

\section{Authors}

Jianwei Qin, Fartash Vasefi, Rosalee S. Hellberg, Alireza Akhbardeh, Rachel B. Isaacs, Ayse Gamze Yilmaz, Chansong Hwang, Insuck Baek, Walter F. Schmidt, and Moon S. Kim 
1 Detection of fish fillet substitution and mislabeling using multimode hyperspectral

3 Jianwei Qin $^{\mathrm{a}}$, Fartash Vasefi ${ }^{\mathrm{b}}$, Rosalee S. Hellberg ${ }^{\mathrm{c}}$, Alireza Akhbardeh ${ }^{\mathrm{b}}$, Rachel B.

4 Isaacs $^{\mathrm{c}}$, Ayse Gamze Yilmaz ${ }^{\mathrm{c}}$, Chansong Hwang ${ }^{\mathrm{a}}$, Insuck Baek ${ }^{\mathrm{a}}$, Walter F. Schmidt ${ }^{\mathrm{a}}$, Moon S. Kim ${ }^{\text {a* }}$

$6 \quad{ }^{a}$ USDA/ARS Environmental Microbial and Food Safety Laboratory, Beltsville

7 Agricultural Research Center, 10300 Baltimore Ave., Beltsville, MD 20705, USA

$8 \quad$ b SafetySpect Inc., 10100 Santa Monica Blvd., Suite 300, Los Angeles, CA 90067, USA

$9 \quad{ }^{\mathrm{c}}$ Schmid College of Science and Technology, Food Science Program, Chapman

10 University, 1 University Drive, Orange, CA 92866, USA

11 * Corresponding author: Moon S. Kim, USDA/ARS/EMFSL, Bldg. 303, BARC-East,

1210300 Baltimore Ave., Beltsville, MD 20705-2350, USA; phone: 1-301-504-8462; fax:

13 1-301-504-9466; e-mail: moon.kim@usda.gov.

15 Abstract: Substitution of high-priced fish species with inexpensive alternatives and 16 mislabeling frozen-thawed fish fillets as fresh are two important fraudulent practices of 17 concern in the seafood industry. This study aimed to develop multimode hyperspectral 18 imaging techniques to detect substitution and mislabeling of fish fillets. Line-scan 19 hyperspectral images were acquired from fish fillets in four modes, including reflectance 20 in visible and near-infrared (VNIR) region, fluorescence by $365 \mathrm{~nm}$ UV excitation, 21 reflectance in short-wave infrared (SWIR) region, and Raman by $785 \mathrm{~nm}$ laser excitation.

22 Fish fillets of six species (i.e., red snapper, vermilion snapper, Malabar snapper, summer

23 flounder, white bass, and tilapia) were used for species differentiation and frozen-thawed 
24 red snapper fillets were used for freshness evaluation. All fillet samples were DNA tested

25 to authenticate the species. A total of 24 machine learning classifiers in six categories (i.e.,

26 decision trees, discriminant analysis, Naive Bayes classifiers, support vector machines, k-

27 nearest neighbor classifiers, and ensemble classifiers) were used for fish species and

28 freshness classifications using four types of spectral data in three different datasets (i.e.,

29 full spectra, first ten components of principal component analysis, and bands selected by

30 sequential feature selection method). The highest accuracies were achieved at $100 \%$ using

31 full VNIR reflectance spectra for the species classification and $99.9 \%$ using full SWIR

32 reflectance spectra for the freshness classification. The VNIR reflectance mode gave the

33 overall best performance for both species and freshness inspection, and it will be further

34 investigated as a rapid technique for detection of fish fillet substitution and mislabeling.

35 Keywords: Hyperspectral imaging; fish mislabeling; reflectance; fluorescence; Raman;

36 machine learning.

\section{1. Introduction}

39 Fish authentication is a major concern for consumers, government agencies and the

40 seafood industry. With increased global trade of fish, complex supply chains, and limited

41 monitoring, there is a rising vulnerability for fish fraud in the marketplace. A large-scale

42 survey by the nonprofit organization Oceana found that $21 \%$ of fish sold in fish markets,

43 grocery stores, and restaurants across the United States was mislabeled on the basis of

44 species (Warner, Roberts, Mustain, Lowell, \& Swain, 2019). Additional forms of

45 mislabeling include labeling frozen-thawed fish as "fresh", misrepresentation of

46 production method (farmed-raised/wild-caught, organic/conventional), and falsification of 
47 geographical origin. Fish mislabeling is a form of economic deception, and also removes

48 the ability for customers to make informed purchases based on conservation management

49 practices for specific populations as well as potential health risks involved with certain fish

50 (e.g., presence of heavy metals, toxins and antibiotic residues). After removing

51 morphological indicators such as heads, tails, skins, and fins, many fish fillets are similar

52 in appearance, which makes them a vulnerable target for economically-motivated fraud. In

53 order to avoid economic deception, there is a need for rapid detection technologies for fish

54 mislabeling and substitution that can be used onsite by seafood importers and distributors.

55 These technologies would serve to improve the assessment of fish quality and

56 authentication to meet the expectations of consumers.

57 Current techniques for detecting fish species with missing taxonomic features are 58 mainly based on molecular methods (Hellberg \& Morrissey, 2011). DNA barcoding is

59 commonly used to identify fish species and it has been adopted by the U.S. Food and Drug

60 Administration for testing regulatory fish samples (Handy, Deeds, Ivanova, Hebert, Hanner,

61 Ormos, \& Yancy, 2011). The DNA sequencing-based technique provides accurate

62 identification of species through comparative analysis of sequence variation in a short

63 fragment of the genome against an existing library of reference sequences (Hebert,

64 Cywinska, Ball, \& deWaard, 2003). But the entire process typically requires 1-2 days of

65 laboratory work and data analysis to identify the species of a given sample. Hence this

66 method is not utilized onsite at processing facilities. Real-time PCR is a rapid method for

67 species identification that is increasingly portable (Naaum, Hellberg, Okuma, \& Hanner,

68 2019); however, it is a targeted method and cannot be used to simultaneously test for a

69 wide range of species. Besides the molecular methods, traditional methods (e.g., 
70 physicochemical analysis, sensory analysis, rheological methods, and electrical

71 measurements) have also been used to evaluate fish and other seafoods (Hassoun \& Karoui,

72 2017). Despite high accuracies of these methods, they generally need expensive and

73 complicated instruments and time-consuming sample preparation procedures, which

74 prevents them from being used for rapid and high-throughput assessment of the aquatic 75 products.

76 Optical sensing techniques (e.g., spectroscopy and imaging) have been developed

77 for quality evaluation of whole fish and fish fillet, which provide a simple, fast, low-cost,

78 and nondestructive alternative to the conventional methods. Various spectroscopy

79 techniques have been investigated, such as visible (VIS), near-infrared (NIR), mid-infrared

80 (MIR), fluorescence, Raman, impedance, and nuclear magnetic resonance (NMR) (Ghidini

$81 \&$ Zanardi, 2019). Example spectroscopy applications for fish include classification of fish

82 species using NIR (Grassi, Casiraghi, \& Alamprese, 2018), Raman (Rašković, Heinke,

83 Rösch, \& Popp, 2016), and NMR spectroscopy (Standal, Axelson, \& Aursand, 2010),

84 evaluation of fish freshness using VIS-NIR (Uddin, Okazaki, Turza, Yumiko, Tanaka, \&

85 Fukuda, 2005), fluorescence (Karoui, Thomas, \& Dufour, 2006), MIR (Karoui, Lefur,

86 Grondin, Thomas, Demeulemester, De Baerdemaeker, \& Guillard, 2007), Raman

87 (Velioğlu, Temiz, \& Boyaci, 2015), and impedance spectroscopy (Fuentes, Masot,

88 Fernández-Segovia, Ruiz-Rico, Alcañiz, \& Barat, 2013), differentiation of farmed-raised

89 and wild-caught fish using NIR (Ottavian, Facco, Fasolato, Novelli, Mirisola, Perini, \&

90 Barolo, 2012) and NMR spectroscopy (Rezzi, Héberger, Axelson, Moretti, Reniero, \&

91 Guillou, 2007), and identification of geographical origin of fish using NIR (Liu, Ma, Wang,

92 Liu, Fan, \& Cao, 2015) and NMR spectroscopy (Aursand, Standal, Praél, Mcevoy, Irvine, 
$93 \&$ Axelson, 2009). External appearance of the whole fish (e.g., shape, color, and texture)

94 has been utilized for species identification using machine vision and image processing

95 techniques (Hu, Li, Duan, Han, Chen, \& Si, 2012; White, Svellingen, \& Strachan, 2006).

96 Hyperspectral imaging (HSI) techniques have become a powerful tool to inspect

97 food and agricultural products (Qin, Kim, Chao, Chan, Delwiche, \& Cho, 2017), and they

98 have been used for quality analysis of fish and other seafoods (Cheng \& Sun, 2014).

99 Example HSI applications for fish include mapping of fat and water content distribution

100 (ElMasry \& Wold, 2008), differentiation of fresh and frozen-thawed fish fillets (Cheng,

101 Sun, Pu, Chen, Liu, Zhang, \& Li, 2015a; Zhu, Zhang, He, Liu, \& Sun, 2013), determination

102 of microbial (Wu \& Sun, 2013) and chemical spoilage (Cheng, Sun, Pu, \& Zhu, 2015b),

103 inspection of blood in fish muscle (Skjelvareid, Heia, Olsen, \& Stormo, 2017), and

104 detection of microplastics in intestinal tracts of fish (Zhang, Wang, Shan, Zhao, Zhang,

105 Liu, \& $\mathrm{Wu}, 2019)$. To our knowledge, reflectance measurement is the only hyperspectral

106 imaging mode used for fish applications in the published studies, and it has been mainly

107 carried out in visible and near-infrared (400-1000 nm) and near-infrared (900-1700 nm)

108 wavelength ranges. Other HSI modes have not been explored, although the equivalent

109 spectroscopy techniques (e.g., fluorescence and Raman) have demonstrated promising

110 results for inspection of fish products.

111 This study aimed to investigate the potential of multimode hyperspectral imaging

112 techniques, including reflectance, fluorescence, and Raman, to detect substitution and

113 mislabeling of fish fillets. Specific objectives were to: (1) collect multimode hyperspectral

114 images from fish fillets of different species and different freshness conditions and (2)

115 develop spectral processing and machine learning classification methods and compare their 
116 performances to differentiate fish species and evaluate fish freshness.

\section{2. Materials and methods}

\section{2.1. Multimode hyperspectral imaging systems}

120 Three in-house developed line-scan hyperspectral imaging systems were used to

121 collect four types of image data from fish fillet samples: (1) reflectance images in visible 122 and near-infrared (VNIR) region, (2) fluorescence images by $365 \mathrm{~nm}$ UV excitation, (3)

123 reflectance images in short-wave infrared (SWIR) region, and (4) Raman images by 785

$124 \mathrm{~nm}$ laser excitation. Major components of the hyperspectral systems and parameters used 125 for image acquisitions are summarized in Table 1.

126 A VNIR hyperspectral system (Kim, Chao, Chan, Jun, Lefcourt, Delwiche, Kang, $127 \&$ Lee, 2011) was used to acquire both reflectance and fluorescence images. A $150 \mathrm{~W}$ 128 quartz tungsten halogen lamp (Dolan Jenner, Boxborough, MA, USA) was used as the 129 illumination source for reflectance imaging. The light was transported from the lamp 130 enclosure via an optic fiber assembly to form two thin line lights that were arranged parallel 131 to the transverse direction. In addition, two UV line lights, each with four $10 \mathrm{~W} 365 \mathrm{~nm}$ 132 light-emitting diodes (LEDs) (LedEngin, San Jose, CA, USA), were used for fluorescence 133 imaging. The detection unit consisted of a $23 \mathrm{~mm}$ focal length lens, an imaging 134 spectrograph (Hyperspec-VNIR, Headwall Photonics, Fitchburg, MA, USA), and a 14-bit 135 electron-multiplying charge-coupled-device (EMCCD) camera (Luca DL 604M, Andor

136 Technology, South Windsor, CT, USA). The reflectance and fluorescence images were 137 acquired in spectral regions of 419-1007 nm (125 bands) and 438-718 nm (60 bands), 138 respectively. 
140 to acquire reflectance images in the SWIR region. The illumination was provided by a 141 custom-designed two-unit lighting system, each with four $150 \mathrm{~W}$ gold-coated halogen

142 lamps with MR16 reflectors. The detection unit included a $25 \mathrm{~mm}$ focal length lens and a

143 hyperspectral camera including a 16-bit mercury cadmium telluride (MCT) array detector 144 and an imaging spectrograph (Hyperspec-SWIR, Headwall Photonics, Fitchburg, MA,

145 USA). The SWIR reflectance images were acquired in a wavelength range of 842-2532 $146 \mathrm{~nm}(287$ bands).

147 Raman images were acquired by a line-scan hyperspectral Raman system (Qin, 148 Chao, Cho, Peng, \& Kim, 2014). The system used a $30 \mathrm{~W} 785 \mathrm{~nm}$ line laser (OptiGrate,

149 Oviedo, FL, USA) as the excitation source. A $45^{\circ} 785 \mathrm{~nm}$ dichroic beamsplitter was used 150 to project the laser normally on the sample surface, on which the laser line was 151 approximately $200 \mathrm{~mm}$ long and $2 \mathrm{~mm}$ wide. The detection unit consisted of two $785 \mathrm{~nm}$ 152 long-pass filters to block Rayleigh and anti-Stokes scattering signals, a $23 \mathrm{~mm}$ focal length 153 lens, a Raman imaging spectrograph (ImSpector R10E, Specim, Oulu, Finland), and a 16154 bit CCD camera (iKon-M 934, Andor Technology, South Windsor, CT, USA). The system 155 covered a wavenumber range of $103-2831 \mathrm{~cm}^{-1}$ ( 846 bands) with a spectral resolution of $15614 \mathrm{~cm}^{-1}$.

\section{2.2. Experimental samples and procedures}

159 Four fish fillets labeled as "snapper", "flounder", "white bass", and "tilapia" were 160 purchased from a local seafood market in Jessup, MD, USA. In addition, a total of 10 fish 161 fillets labeled as "red snapper" were purchased from three online retailers. Red snapper 
162 (Lutjanus campechanus) was used because it is a high-priced species and one of the most

163 mislabeled fish in the United States (Warner, Roberts, Mustain, Lowell, \& Swain, 2019).

164 Other species were selected since they are commonly mislabeled as red snapper for higher

165 retail prices. All 14 fillets were used for the fish species differentiation study. The fish

166 freshness evaluation study was limited to the red snapper fillets authenticated with DNA

167 barcoding (described in Section 2.3). The fillet samples were transported with ice packs to

168 the USDA/ARS Environmental Microbial and Food Safety Laboratory and they were

169 imaged immediately using the three aforementioned hyperspectral systems under a room

170 temperature of $\sim 20{ }^{\circ} \mathrm{C}$. After imaging, the red snapper fillets were frozen in a $-20{ }^{\circ} \mathrm{C}$

171 freezer for $24 \mathrm{~h}$ and then thawed in a $4{ }^{\circ} \mathrm{C}$ refrigerator for $24 \mathrm{~h}$. The frozen-thawed samples

172 were reimaged using the same three systems. The same freezing and thawing process was

173 repeated for a second cycle, and the samples were imaged for the third time to finish the

174 data acquisition. As a result, three sets of the hyperspectral images were collected from

175 each red snapper fillet, including an "as received" (AR) image and two images

176 corresponding to the two freeze-thaw cycles (FT1 and FT2).

177 Each fillet was placed in a sample holder with a volume of $150 \times 100 \times 25 \mathrm{~mm}^{3}$. For

178 the reflectance and fluorescence measurements, the sample holders were created by a 3D

179 printer (Fortus 250mc, Stratasys, Eden Prairie, MN, USA) using production-grade black

180 thermoplastic. For the Raman measurement, nickel plated aluminum containers were used

181 to minimize signals from the sample holder. In each line-scan hyperspectral system, a linear

182 motorized translation stage was used to move the sample incrementally across the scanning

183 line of the imaging spectrograph, by which the system conducted image acquisition using

184 a push-broom method. The lens-to-sample distance in each system was adjusted so that the 
185 length of the instantaneous field of view (IFOV) of the camera was slightly longer than the

186 length of the sample holder $(150 \mathrm{~mm})$. Under these settings, the spatial resolutions along

187 the IFOV direction of all three systems were determined as $0.4 \mathrm{~mm} / \mathrm{pixel}$. Each fillet

188 sample was scanned along the width direction $(100 \mathrm{~mm})$ of the sample holder using an

189 incremental size of $0.4 \mathrm{~mm}$ to match the spatial resolution of the length direction.

$191 \quad$ 2.3. Fish species authentication with DNA barcoding

192 All fillet samples were DNA tested for species authentication. Before imaging, a

193 small piece of sample ( $\sim 5 \mathrm{~g})$ was removed from the interior of each fillet using a disposable

194 scalpel and sterile forceps and then placed in a $50 \mathrm{~mL}$ sterile Falcon tube. The samples

195 were immediately frozen at $-80{ }^{\circ} \mathrm{C}$ for $24 \mathrm{~h}$ and then shipped overnight with ice to

196 Chapman University for DNA-based identification. DNA was extracted from $\sim 10 \mathrm{mg}$ of

197 each sample using the DNeasy Blood and Tissue Kit (Qiagen, Germantown, MD, USA),

198 Spin-Column protocol, with modifications described in Handy, Deeds, Ivanova, Hebert,

199 Hanner, Ormos, \& Yancy (2011). All samples were lysed with a ThermoMixer C

200 (Eppendorf, Hamburg, Germany) and DNA was eluted using $100 \mu \mathrm{l}$ AE buffer (Qiagen).

201 A reagent blank negative control was included with each set of DNA extractions. After

202 extraction, the DNA in each sample was quantified using a Biophotometer Plus 203 (Eppendorf).

204 Full DNA barcoding of each DNA extract was performed as described in Moore, 205 Handy, Haney, Pires, Perry, Deeds, \& Yancy (2012). Samples that failed to be identified 206 with full barcoding underwent mini-barcoding with the SH-E mini-barcode primers 207 (Shokralla, Hellberg, Handy, King, \& Hajibabaei, 2015) using the following reaction 
208 mixture: 0.5 OmniMix ${ }^{\circledR}$ HS Lyophilized PCR Master Mix bead (Cepheid, Sunnyvale, CA,

209 USA), $22.5 \mu 1$ molecular-grade water, $0.50 \mu 1$ each primer, and $2.0 \mu 1$ DNA. The cycling

210 conditions for mini-barcoding were as described in Shokralla, Hellberg, Handy, King, \&

211 Hajibabaei (2015). Integrated DNA Technologies (Coralville, IA, USA) synthesized all

212 primers. Each set of reactions included a no-template control (NTC) with molecular-grade

213 water in place of DNA. A Mastercycler nexus Gradient Thermal Cycler (Eppendorf) was

214 used for PCR.

215 PCR products were confirmed using 2.0\% agarose E-Gels run on an E-Gel iBase

216 (Invitrogen, Carlsbad, CA, USA) as described in Hellberg, Isaacs, \& Hernandez (2019).

217 All samples with confirmed PCR products were purified with ExoSAP-IT (Affymetrix,

218 Santa Clara, CA, USA), then shipped to the GenScript facility (Piscataway, NJ, USA) for

219 DNA sequencing. DNA sequences were assembled and edited using Geneious R7

220 (Biomatters, Auckland, New Zealand) with quality parameters described in Pollack,

221 Kawalek, Williams-Hill, \& Hellberg (2018). Consensus sequences were identified based

222 on the top species match in the Barcode of Life Database (BOLD) Animal Identification

223 Request Engine (http://www.boldsystems.org/), Full Length Published Records.

\section{2.4. Spectral and image processing and machine learning classifications}

226 Fig. 1 summarizes the general data analysis procedures. Flat-field corrections were

227 conducted on VNIR and SWIR reflectance images to convert original intensities in CCD

228 counts to relative reflectance in percent. Similar corrections were also used for fluorescence

229 images to obtain the relative fluorescence intensities (Kim, Chen, \& Mehl, 2001).

230 Fluorescence background in Raman images was removed by a baseline correction method 
231 using adaptive iteratively reweighted penalized least squares (Zhang, Chen, \& Liang, 2010).

232 After preprocessing the four types of the hyperspectral images, a single-band image was

233 selected for each sample at a wavelength/wavenumber $\left(\lambda_{\mathrm{m}}\right)$ with the maximum spectral

234 intensity (i.e., VNIR reflectance, fluorescence, SWIR reflectance, or Raman) of the fish

235 fillet, which was used to create a spatial mask to remove the sample background. Then all

236 the fish pixels in the masked image at $\lambda_{\mathrm{m}}$ were grouped into $10 \times 10$ pixel windows to mimic

237 point spectroscopy measurements. The mean (M) and standard deviation (STD) of the fish

238 pixel intensities within each window were calculated and evaluated to remove regions with

239 large variations. When $10 \%$ of the 100 pixels were beyond the range of $\mathrm{M} \pm 2 \mathrm{STD}$, the

240 whole pixel window was excluded for further analysis. The 100 spectra extracted from

241 each remaining window were averaged in the spatial domain while the full spectral

242 resolution was maintained. All the mean spectra were used for machine learning

243 classifications. This segmentation method generated four types of point spectral datasets

244 for developing algorithms that can be adopted for future low-cost point spectroscopy

245 systems for fish authentication.

246 The four types of the spectral data were labeled using the DNA test results for the

247 fish species and the freshness status for the red snapper fillets. The labeled data were input

248 to the Classification Learner app in MATLAB (R2019a, MathWorks, Natick, MA, USA)

249 to determine how well each spectral measurement can contribute to fish species and

250 freshness evaluation. To reduce data dimensions and improve computational efficiencies,

251 principal component analysis (PCA) and sequential feature selection (SFS) functions in

252 MATLAB were used as feature extraction and selection methods respectively to create two

253 subsets. One subset was the first ten components of the PCA and the other was a subset 
254 with significant bands for each of the four types of the images. The SFS algorithm

255 identified the bands that best classified fish species or freshness by sequentially selecting

256 important features and removing irrelevant features until there was no improvement for the

257 classification accuracy. The full spectra, the first ten PCA components, and the spectral

258 data at selected bands were all used for the machine learning classifications, and the

259 accuracies using the three datasets were compared.

260 A total of 24 classifiers in six general categories (i.e., decision trees, discriminant

261 analysis, Naive Bayes classifiers, support vector machines (SVMs), k-nearest neighbor

262 (KNN) classifiers, and ensemble classifiers) were tested to assess the classification

263 performance for each type of the spectral data. To simplify the evaluation of

264 misclassification costs and model training, equal penalty was assigned to all species and

265 freshness misclassifications and default hyperparameters in the MATALB Classification

266 Learner app (e.g., maximum number of splits for a decision tree, box constraint level of an

267 SVM, and distance metric of a KNN) were used for all 24 preset classifiers. Although the

268 default hyperparameters may not be optimized for all the classifiers, they saved the training

269 time and provided a quick and direct approach to compare accuracies of the different

270 models, which was consistent with the purpose of this pilot study. Given the large sample

271 sizes ( $\geq 5129$ spectra in each classification, see Tables 2 and 3), two-fold cross-validation

272 was used to minimize the overfitting problem and evaluate the generalization abilities and

273 predictive accuracies of all the classification models. Each spectral dataset was randomly

274 partitioned into two equal-size disjoint folds. A model was trained using out-of-fold data

275 and the performance was assessed using in-fold data. The two folds were used as

276 independent datasets for training and validation, respectively, which was conducted with a 
277 goal of minimizing the classification error. The overall accuracy of each model was

278 obtained by calculating the average error over the two folds. Details for the classification

279 algorithms and hyperparameters can be found in MathWorks (2019).

281 3. Results and discussion

282 3.1. DNA test results

283 The four fish fillets labeled by the local seafood market as "snapper", "flounder",

284 "white bass", and "tilapia" were identified by DNA barcoding as vermilion snapper

285 (Rhomboplites aurorubens), summer flounder (Paralichthys dentatus), white bass (Morone

286 chrysops), and tilapia (Oreochromis sp.), respectively. The DNA tests also confirmed that

287 six "red snapper" fillets purchased from two online retailers (three from each) were

288 correctly labeled. However, four fillets labeled by one online retailer as "red snapper" were

289 identified as Malabar snapper (Lutjanus malabaricus), which was a real-life fish

290 mislabeling case occurred during sample collections in this study. The samples identified

291 as red snapper, vermilion snapper, Malabar snapper, summer flounder, white bass, and

292 tilapia were used for the species classification study (Fig. 2a), and the six authenticated red

293 snapper fillets were used for the freshness classification study. Fig. 2b shows an example

294 red snapper fillet as received and after two freeze-thaw cycles.

296 3.2. Hyperspectral images and spectra of fish fillets

297 Fig. 3 shows four types of hyperspectral images acquired from a red snapper fillet.

298 The four single-band images were extracted from the hyperspectral images at selected

299 spectral peak positions to demonstrate the general pattern of a fish fillet in each imaging 
300 type. The fillet surface appear more consistent in the VNIR and SWIR reflectance images

301 than in the fluorescence and Raman images, revealing that the fluorescence and Raman

302 signals may be more sensitive to the fish tissue variations than the VNIR and SWIR

303 reflectance signals.

304 An example of extracting spectra from the VNIR reflectance image of a red snapper

305 fillet was demonstrated in Fig. 4. The single-band image at $699 \mathrm{~nm}$, at which the fish tissue

306 showed highest reflectance (Fig. 4b), was used to generate a mask image (Fig. 4a) to isolate

307 the fillet from the background. After evaluating the pixel intensity variations for all $10 \times 10$

308 pixel windows in the masked $699 \mathrm{~nm}$ image of the fillet, an average-window image was

309 created, in which the total number of the remaining windows was determined as 463 . Mean

310 reflectance spectra calculated within each of the 463 windows are plotted in Fig. $4 \mathrm{~b}$.

311 Mean spectra of red snapper and five other species commonly mislabeled as red

312 snapper are plotted in Fig. 5. The VNIR reflectance spectra (Fig. 5a) show different patterns

313 due to compositional variations of the fillets. The broad reflectance valley at $560 \mathrm{~nm}$ and

314 two small valleys at 546 and $578 \mathrm{~nm}$ likely correspond with the absorption peaks of the

315 heme pigments in the fish tissue, such as hemoglobin in the blood filled vessels and

316 myoglobin in the muscle. The reduced reflectance at $636 \mathrm{~nm}$ is more evident in tilapia, red

317 snapper, vermillion snapper, and moderately in white bass and appears to correspond with

318 methemoglobin absorption regions. Main spectral features of the SWIR reflectance (Fig.

319 5c) appear in the wavelength range of $900-1500 \mathrm{~nm}$, and their spectral patterns exhibit

320 more consistency than the VNIR reflectance spectra. Two major valleys were observed at

321984 and $1208 \mathrm{~nm}$, which are associated with the first O-H stretching overtone of water and 
322 the second $\mathrm{C}-\mathrm{H}$ stretching overtone of fat, respectively. The variations in the SWIR

323 reflectance intensities indicate different fat and water content for the different fish species.

324 The fluorescence spectra (Fig. 5b) show distinctive differences, which could arise

325 from different protein-protein interactions and collagen structures among the various

326 species. It is interesting to find that the fluorescence intensities of red snapper are lower

327 than all other five species in the whole spectral region. Major Raman peaks of the fillet 328 samples can be assignable to the lipid component in the fish, and their vibrational modes

329 and chemical bonds are marked in Fig. 5d. The Raman peaks near 734, 1451, and 1651

$330 \mathrm{~cm}^{-1}$ are characteristic of long chain unsaturated fatty acid components as free acids and/or

331 esters. The peaks near $1311 \mathrm{~cm}^{-1}$ are associated with $\mathrm{C}-\mathrm{O}$ stretching especially in C-O-C

332 moieties, including in $\mathrm{C}-\mathrm{O}-\mathrm{C}=\mathrm{O}$ sites. Wavenumbers of 636 and $1097 \mathrm{~cm}^{-1}$ are consistent

333 with $\mathrm{C}-\mathrm{O}$ stretching as in $\mathrm{C}-\mathrm{O}-\mathrm{H}$ and $\mathrm{O}-\mathrm{H}$ twisting in $\mathrm{C}-\mathrm{O}-\mathrm{H}$ as would be present in free

334 lipid fatty acids. Two peaks near 487 and $2305 \mathrm{~cm}^{-1}$ are attributed to phospholipids

335 glycerol esters including phosphotidylcholines. More complicated vibrational modes

336 between 800 and $1000 \mathrm{~cm}^{-1}$ correspond with out of plane bending of C-H especially

337 adjacent to $\mathrm{C}=\mathrm{C}$ sites. Wavenumbers are different depending on the number of double

338 bonds in the particular lipid of interest. This demonstrates the lipids in the fish can have

339 quite different unsaturated lipid composition.

340 Fig. 6 shows mean spectra of red snapper fillets as received (AR) and after two

341 freeze-thaw (FT) cycles. The overall patterns of the FT fillets are similar to those of the

342 AR fillets for all four types of the spectra. In both VNIR (Fig. 6a) and SWIR (Fig. 6c)

343 regions, the FT fillets exhibit lower reflectance intensities than the AR fillets, whereas the

344 differences between the first (FT1) and the second (FT2) freeze-thaw cycles are not 
345 significant. Such patterns were not observed in the fluorescence (Fig. 6b) and Raman (Fig.

346 6d) spectra. Instead, the fluorescence and Raman spectra of the FT2 samples show some

347 intensity changes from the AR and FT1 samples, and there is little difference between the

348 AR and FT1 samples. The four types of the spectral signals can be affected by a broad

349 range of factors, such as fish tissue damage, texture deterioration, protein denaturation,

350 water holding capacity, muscle toughening, and lipid and heme pigment oxidation (Zhu,

351 Zhang, He, Liu, \& Sun, 2013). Previous studies on halibut (Zhu, Zhang, He, Liu, \& Sun,

352 2013) and grass carp (Cheng, Sun, Pu, Chen, Liu, Zhang, \& Li, 2015a) found that frozen-

353 thawed fillets had higher reflectance than fresh fillets in the VNIR region, which is opposite

354 the trend observed in the current study for reflectance measurements on the red snapper

355 fillets. One possible reason is that during the freezing and thawing process, the red snapper

356 generated more oxidized heme pigments than other fish species. The oxidized heme

357 pigments would have resulted in a darker color and thus reduced reflectance for the frozen-

358 thawed red snapper samples. In this pilot study, we have not tested species other than red

359 snapper for the effects of the freezing and thawing process on the spectral measurements.

360 It remains for further investigation to ascertain whether the reflectance, fluorescence, and

361 Raman spectral differences found in this study are consistent with other fish species and

362 other variations of the freeze-thaw cycles.

363 The bands selected by the SFS method for species and freshness classifications are

364 marked in Fig. 5 and Fig. 6, respectively. For VNIR and SWIR reflectance spectra, the

365 selected bands are mainly located in separated spectral absorption regions. All VNIR bands

366 selected for the species classification are in the heme pigment absorption region (Fig. 5a).

367 Three bands near water absorption were selected for the freshness classification in addition 
368 to the three bands near heme pigment absorption (Fig. 6a). The SWIR bands selected for

369 both species (Fig. 5c) and freshness (Fig. 6c) classifications are close to the water and fat

370 absorption areas, except that one band was selected in the flat reflectance range near 2300

$371 \mathrm{~nm}$. On the other hand, the selected bands in the fluorescence (Figs. 5b and 6b) and Raman

372 (Figs. 5d and 6d) spectra are generally spread over the whole wavelength ranges. The bands

373 were selected at spectral peaks, valleys, shoulders, and some flat baseline regions. These

374 results suggest that the bands selected by the SFS method may or may not be directly linked

375 to the physical features reflected by each type of the spectral data.

376

377 3.3. Fish species classifications

$378 \quad$ Numbers of mean spectra extracted from hyperspectral images of 14 fillet samples

379 for species classifications are summarized in Table 2. Fig. 7 presents confusion matrices

380 generated from the species classifications using linear SVM classifier with four types of

381 the full spectral data. The correctly classified instances and true positive rates are marked

382 in the diagonal of each matrix, whereas the misclassified instances and false negative rates

383 are marked in the shaded grids outside the diagonal. The confusion matrices provide a

384 visualization for the classification performance of each spectral data type and can help

385 understand which species can be most easily confused using each of the spectral

386 measurement modes. For the VNIR reflectance (Fig. 7a), tilapia and vermillion snapper

387 were misclassified as red snapper with relatively high percentages $(8.4 \%$ and $4.2 \%$,

388 respectively). There was no pattern of high misclassification for the fluorescence data (Fig.

$3897 \mathrm{~b}$ ) considering all individual false negative rates were no larger than $1.2 \%$. The SWIR

390 reflectance spectra (Fig. 7c) had high false classifications $(\geq 4.5 \%)$ for all the species except 
391 for tilapia (0.9\%). The highest false negative rate occurred for Malabar snapper, as $19.1 \%$

392 were misclassified as red snapper. Also, all five non-tilapia species were misclassified as

393 tilapia with relatively high percentages (4.5-7.5\%). Similar to the fluorescence data,

394 individual false negative rates for the Raman spectra (Fig. 7d) were no larger than $1.2 \%$,

395 with one exception that $5.6 \%$ of Malabar snapper was misclassified as red snapper. For this

396 particular example using the linear SVM classifier and the full spectral data, the overall

397 classification accuracy was highest for fluorescence (99.4\%), followed by VNIR

398 reflectance (98.5\%), Raman (97.6\%), and SWIR reflectance (88.2\%). Note that the

399 discussions above were mainly based on the true positive and false negative rates as well

400 as the overall accuracies. Other classification performance measures, such as positive

401 predictive values (precisions) and false discovery rates (not used in this study), can also be

402 calculated using the numbers of observations in the confusion matrices.

$403 \quad$ Fig. 8 summarizes fish species classification results by 24 machine learning 404 classifiers using four types of spectral data in three different datasets (i.e., full spectra, first 405 ten components of PCA, and bands selected by SFS). Each data point in the figure is an 406 overall accuracy for classifying the six fish species. As shown in the figure, different 407 combinations of classifier, spectral type, and dataset result in different classification 408 accuracies, which can help visualize the general trend and identify the best combination. 409 For the full spectra (Fig. 8a), the VNIR reflectance data achieved two perfect classifications 410 (100\% accuracy) using linear discriminant and subspace discriminant classifiers. Linear, 411 quadratic, and cubic SVMs gave high accuracies (97.6-99.5\%) for the VNIR reflectance, 412 fluorescence, and Raman data. Naive Bayes classifiers yielded the worst results $(<80 \%)$ 413 for all four types of the spectra. The accuracies using the PCA data (Fig. 8b) and the 
414 selected bands (Fig. 8c) exhibited some similar patterns with those using the full spectra.

415 High accuracies (98.1-100\%) were also obtained for the VNIR reflectance and

416 fluorescence data using the linear, quadratic, and cubic SVMs. Overall, the VNIR

417 reflectance and fluorescence data provided the best performance for classifying the fish

418 species. The accuracies using the Raman data were slightly lower and the SWIR reflectance

419 data generally gave the lowest accuracies. These results can be attributed to the fact that

420 spectral differences among the six fish species for the VNIR reflectance and fluorescence

421 data are generally larger than those of the Raman and SWIR reflectance data (see Fig. 5).

\section{3.4. Fish freshness classifications}

$424 \quad$ Table 3 lists numbers of mean spectra extracted from hyperspectral images of six 425 red snapper fillets for freshness classifications. The confusion matrices for classifying red 426 snapper freshness using the linear SVM classifier with four types of the full spectral data 427 are shown in Fig. 9. In VNIR reflectance (Fig. 9a) and Raman (Fig. 9d) datasets, 428 classification was more accurate when the fish fillet underwent two freeze-thaw cycles 429 compared to one cycle. For VNIR, Raman, and fluorescence (Fig. 9b), the as-received (AR)

430 fillets were more easily misclassified as frozen-thawed fillets in the first cycle (FT1) rather 431 than those in the second cycle (FT2). Also, for the VNIR and fluorescence data the FT1 432 and FT2 samples tended to be misclassified as each other rather than as the AR samples.

433 This is important because it suggests there is a progressive change in the fish tissue 434 associated with the freeze-thaw process. In this pilot study, we have not undertaken more 435 detailed comparisons for the duration and other variations of the freeze-thaw cycles. 436 However, future research to explore the effects of these variations will be carried out. 
437 Interestingly, the SWIR reflectance spectra (Fig. 9c) did not show the same progressive

438 trend associated with freeze-thaw cycles observed for the VNIR and fluorescence data. A

439 small portion of the AR samples (1.9\%) were misclassified as the FT2 samples but not the

440 FT1 samples. Also, the FT1 and FT2 samples were both misclassified as the AR samples

441 without any misclassification among each other. The Raman results (Fig. 9d) showed a

442 similar confusion pattern with those of the VNIR reflectance and fluorescence data, with

443 one exception that the percentage of the FT1 misclassified as the AR (30.4\%) was much

444 higher than that of the FT1 misclassified as the FT2 (7.1\%). For the example shown in Fig.

4459 , the overall classification accuracy was highest for SWIR reflectance $(95.5 \%)$ and VNIR

446 reflectance (95.0\%), followed by fluorescence (90.1\%) and Raman (74.4\%).

447 The freshness classification results for the red snapper fillets are summarized in Fig.

448 10. For the full spectra (Fig. 10a), the highest classification accuracy was $99.9 \%$, which

449 was achieved by the subspace discriminant classifier on the SWIR reflectance data. High

450 accuracies (98.1-99.0\%) were also obtained for the VNIR reflectance data when the linear

451 and quadratic discriminant classifiers and the quadratic and cubic SVMs were used. The

452 first ten components of PCA for the VNIR reflectance spectra generally gave higher

453 accuracies than the other three types of the spectra for most of the 24 classifiers (Fig. 10b),

454 with the highest accuracy obtained by the cubic SVM at $97.4 \%$. The accuracies using the

455 selected bands from the VNIR reflectance and fluorescence spectra (Fig. 10c) were

456 generally lower than those using the full spectra and the PCA data. The SWIR reflectance

457 data outperformed the other three types of the data even only three bands were selected for

458 the classifications (see Fig. 6c), with the highest accuracy obtained by the quadratic SVM

459 at $95.3 \%$. Regardless of the classifiers and the datasets, the performance of the fluorescence 
460 spectroscopy was moderate, and the Raman data generally yielded the lowest accuracies

$461(<80 \%)$. These results demonstrated that the VNIR and SWIR reflectance modes seem

462 more suitable for the fish freshness classification than the fluorescence and Raman modes.

463 Water content change in the fish tissue is associated with the freezing and thawing process

464 of the fillet samples. Both fluorescence and Raman signals have low sensitivity to changes

465 in water content, which might be a reason for the relatively low classification accuracies

466 for the two spectroscopy techniques.

467 Considering fish species and freshness classifications together, the VNIR

468 reflectance spectroscopy technique coupled with selected machine learning classifiers (e.g.,

469 discriminant analysis and SVM classifiers) demonstrated strong performance for both tasks.

470 The next steps in this research will be to investigate the method further using a greater

471 range of fish species and additional variations of the freeze-thaw cycles. Meanwhile,

472 designing and building customized VNIR reflectance spectroscopy and imaging systems

473 (e.g., handheld detection devices and online hyperspectral systems) suitable for industrial

474 fish inspection applications are also planned.

475

\section{4. Conclusion}

477 This study presented multimode hyperspectral imaging techniques to inspect 478 substitution and mislabeling for fish fillets. Four types of spectra (i.e., reflectance in visible

479 and near-infrared region, fluorescence, reflectance in short-wave infrared region, and

480 Raman) extracted from hyperspectral images of the fish fillets created sufficiently large 481 datasets to train and validate machine learning classifiers for fish species and freshness 482 classifications. Results from different combinations of machine learning classifier, spectral 
483 type, and dataset provided an intuitive way to compare their performances and identify the

484 best combination. The highest classification accuracies were achieved using selected 485 machine learning classifiers to differentiate the fish species and evaluate the fish freshness

486 using full reflectance spectra in the visible and near-infrared region and the short-wave 487 infrared region, respectively. The reduced spectral datasets by principal component 488 analysis and sequential feature selection methods generally yielded lower classification 489 accuracies than the full datasets. The reflectance spectroscopy technique in visible and 490 near-infrared region demonstrated its potential for simultaneous inspection of the fish 491 species and freshness. This technique has high potential to be utilized in a low-cost point 492 spectroscopy device for real-time authentication of the fish fillets. Future work will be

493 conducted to validate the method using more fish species and additional variations of the 494 freeze-thaw cycles. Alternative feature extraction and selection methods and 495 hyperparameter optimization for the classification models will also be tested for future 496 larger datasets.

\section{References}

499 Aursand, M., Standal, I. B., Praél, A., Mcevoy, L., Irvine, J., \& Axelson, D. E. (2009).

$500 \quad{ }^{13} \mathrm{C}$ NMR pattern recognition techniques for the classification of Atlantic salmon 501 (salmo salar L.) according to their wild, farmed, and geographical origin. Journal 502 of Agricultural and Food Chemistry, 57, 3444-3451.

$503 \quad$ https://doi.org/10.1021/jf8039268 
504 Cheng, J. \& Sun, D. (2014). Hyperspectral imaging as an effective tool for quality analysis and control of fish and other seafoods. Trends in Food Science \& Technology, 37(2), 78-91. https://doi.org/10.1016/j.tifs.2014.03.006

507 Cheng, J., Sun, D., Pu, H., Chen, X., Liu, Y., Zhang, H., \& Li, J. (2015a). Integration of classifiers analysis and hyperspectral imaging for rapid discrimination of fresh

Cheng, J., Sun, D., Pu, H., \& Zhu, Z. (2015b). Development of hyperspectral imaging from cold-stored and frozen-thawed fish fillets. Journal of Food Engineering, 161, 33-39. https://doi.org/10.1016/j.jfoodeng.2015.03.011

coupled with chemometric analysis to monitor $K$ value for evaluation of chemical spoilage in fish fillets. Food Chemistry, 185, 245-253.

http://dx.doi.org/10.1016/j.foodchem.2015.03.111

ElMasry, G. \& Wold, J. P. (2008). High-speed assessment of fat and water content distribution in fish fillets using online imaging spectroscopy. Journal of Agricultural and Food Chemistry, 56, 7672-7677. https://doi.org/10.1021/jf801074s

Fuentes, A., Masot, R., Fernández-Segovia, I., Ruiz-Rico, M., Alcañiz, M., \& Barat, J. M. (2013). Differentiation between fresh and frozen-thawed sea bream (Sparus aurata) using impedance spectroscopy techniques. Innovative Food Science and Emerging Technologies, 19, 201-217. http://dx.doi.org/10.1016/j.ifset.2013.05.001

Ghidini, S., Varrà, M. O., \& Zanardi, E. (2019). Approaching authenticity issues in fish 525 and seafood products by qualitative spectroscopy and chemometrics. Molecules, $526 \quad 24,1812$. http://dx.doi.org/10.3390/molecules24091812 
527 Grassi, S., Casiraghi, E., \& Alamprese, C. (2018). Handheld NIR device: A non-targeted

528 approach to assess authenticity of fish fillets and patties. Food Chemistry, 243,

$529 \quad 382-388$. https://doi.org/10.1016/j.foodchem.2017.09.145

530 Handy, S. M., Deeds, J. R., Ivanova, N. V., Hebert, P. D. N., Hanner, R. H., Ormos, A.,

531 \& Yancy, H. F. (2011). A single-laboratory validated method for the generation of

532 DNA barcodes for the identification of fish for regulatory compliance. Journal of 533 AOAC International, 94(1), 201-210.

534 Hassoun, A. \& Karoui, R. (2017). Quality evaluation of fish and other seafood by

535 traditional and nondestructive instrumental methods: Advantages and limitations.

$536 \quad$ Critical Reviews in Food Science and Nutrition, 57(9), 1976-1998.

$537 \quad$ http://dx.doi.org/10.1080/10408398.2015.1047926

538 Hebert, P. D. N., Cywinska, A., Ball, S. L., \& deWaard, J. R. (2003). Biological

539 identifications through DNA barcodes, Proceedings of the Royal Society B:

$540 \quad$ Biological Sciences, 270, 313-321. https://doi.org/10.1098/rspb.2002.2218

541 Hellberg, R. S., Isaacs, R. B., \& Hernandez, E. L. (2019). Identification of shark species

542 in commercial products using DNA barcoding. Fisheries Research, 210, 81-88.

$543 \quad$ https://doi.org/10.1016/j.fishres.2018.10.010

544 Hellberg, R. S. \& Morrissey, M. T. (2011). Advances in DNA-based techniques for the

545 detection of seafood species substitution on the commercial market. Journal of the

$546 \quad$ Association for Laboratory Automation, 16(4), 308-321.

$547 \quad$ https://doi.org/10.1016/j.jala.2010.07.004

548 Hu, J., Li, D., Duan, Q., Han, Y., Chen, G., \& Si, X. (2012). Fish species classification by

549 color, texture and multi-class support vector machine using computer vision. 
552 Karoui, R., Lefur, B., Grondin, C., Thomas, E., Demeulemester, C., De Baerdemaeker, J., \& Guillard, A. S. (2007). Mid-infrared spectroscopy as a new tool for the

Karoui, R., Thomas, E., \& Dufour, E. (2006). Utilisation of rapid technique based on

567 Lee, H., Kim, M. S., Lohumi, S., \& Cho, B. (2018). Detection of melamine in milk powder using MCT-based short-wave infrared hyperspectral imaging system. front-face fluorescence spectroscopy for differentiating between fresh and frozenthawed fish fillets. Food Research International, 39, 349-355. https://doi.org/10.1016/j.foodres.2005.08.007

Kim, M. S., Chao, K., Chan, D. E., Jun, W., Lefcourt, A. M., Delwiche, S. R., Kang, S., \& Lee, K. (2011). Line-scan hyperspectral imaging platform for agro-food safety and quality evaluation: System enhancement and characterization. Transactions of the ASABE, 54(2), 703-711. http://doi.org/10.13031/2013.36473

Kim, M. S., Chen, Y., \& Mehl, P. M. (2001). Hyperspectral reflectance and fluorescence imaging system for food quality and safety. Transactions of the ASAE, 44(3), 721729. http://doi.org/10.13031/2013.6099 Food Additives \& Contaminants: Part A, 35(6), 1027-1037. https://doi.org/10.1080/19440049.2018.1469050

Liu, Y., Ma, D., Wang, X., Liu, L., Fan, Y., \& Cao, J. (2015). Prediction of chemical composition and geographical origin traceability of Chinese export tilapia fillets 
products by near infrared reflectance spectroscopy. LWT Food Science and Technology, 60, 1214-1218. https://doi.org/10.1016/j.lwt.2014.09.009

575 MathWorks. (2019). Statistics and Machine Learning Toolbox User's Guide (Version

57611.5 for MATLAB R2019a). Natick, MA, USA: The MathWorks, Inc.

577 Moore, M. M., Handy, S. M., Haney, C. J., Pires, G. S., Perry, L. L., Deeds, J. R., \&

578 Yancy, H. F. (2012). Updates to the FDA Single Laboratory Validated Method for DNA Barcoding for the Species Identification of Fish. FDA Laboratory Information Bulletin 4528.

581 Naaum, A. M., Hellberg, R. S., Okuma, T. A., \& Hanner, R. H. (2019). Multi-instrument evaluation of a real-time PCR assay for identification of Atlantic salmon: a case study on the use of a pre-packaged kit for rapid seafood species identification.

Ottavian, M., Facco, P., Fasolato, L., Novelli, E., Mirisola, M., Perini, M., \& Barolo, M. Food Analytical Methods, 12, 2474-2479. https://doi.org/10.1007/s12161-019-

Pollack, S. J., Kawalek, M. D., Williams-Hill, D. M., \& Hellberg, R. S. (2018). (2012). Use of near-infrared spectroscopy for fast fraud detection in seafood: Application to the authentication of wild European sea bass (Dicentrarchus labrax). Journal of Agricultural and Food Chemistry, 60, 639-648. Evaluation of DNA barcoding methodologies for the identification of fish species in cooked products. Food Control, 84, 297-304. https://doi.org/10.1016/j.foodcont.2017.08.013 
595 Qin, J., Chao, K., Cho, B., Peng, Y., \& Kim, M. S. (2014). High-throughput Raman

596 chemical imaging for rapid evaluation of food safety and quality. Transactions of

597 the ASABE, 57(6), 1783-1792. http://doi.org/10.13031/trans.57.10862

598 Qin, J., Kim, M. S., Chao, K., Chan, D. E., Delwiche, S. R., \& Cho, B. (2017). Line-scan

599 hyperspectral imaging techniques for food safety and quality applications. Applied

$600 \quad$ Sciences, 7(2), 125. https://doi.org/10.3390/app7020125

601 Rašković, B., Heinke, R., Rösch, P., \& Popp, J. (2016). The Potential of Raman

602 spectroscopy for the classification of fish fillets. Food Analytical Methods, 9,

603 1301-1306. http://dx.doi.org/10.1007/s12161-015-0312-6

604 Rezzi, S., Giani, I., Héberger, K., Axelson, D. E., Moretti, V. M., Reniero, F., \& Guillou,

605 C. (2007). Classification of gilthead sea bream (Sparus aurata) from ${ }^{1} \mathrm{H}$ NMR

606 lipid profiling combined with principal component and linear discriminant

607 analysis. Journal of Agricultural and Food Chemistry, 55, 9963-9968.

$608 \quad$ https://doi.org/10.1021/jf070736g

609 Shokralla, S., Hellberg, R. S., Handy, S. M., King, I., \& Hajibabaei, M. (2015). A DNA

610 mini-barcoding system for authentication of processed fish products. Scientific

611 Reports, 5, 15894. https://doi.org/10.1038/srep15894

612 Skjelvareid, M. H., Heia, K., Olsen, S.H., \& Stormo, S. K. (2017). Detection of blood in

613 fish muscle by constrained spectral unmixing of hyperspectral images. Journal of

$614 \quad$ Food Engineering, 212, 252-261.

$615 \quad$ http://dx.doi.org/10.1016/j.jfoodeng.2017.05.029

616 Standal, I. B., Axelson, D. E., \& Aursand, M. (2010). ${ }^{13}$ C NMR as a tool for

617 authentication of different gadoid fish species with emphasis on phospholipid 
profiles. Food Chemistry, 121, 608-615.

620 Uddin, M., Okazaki, E., Turza, S., Yumiko,Y., Tanaka, M., \& Fukuda,Y. (2005). Non-

621 destructive visible/NIR spectroscopy for differentiation of fresh and frozenthawed fish. Journal of Food Science, 70, C506-C510.

624 Velioğlu, H. M., Temiz, H. T., \& Boyaci, I. H. (2015). Differentiation of fresh and 625 frozen-thawed fish samples using Raman spectroscopy coupled with chemometric analysis. Food Chemistry, 173, 283-290. http://dx.doi.org/10.1016/j.foodchem.2014.09.073

628 Warner, K., Roberts, W., Mustain, P., Lowell, B., \& Swain, M. (2019). Casting a Wider Net: More Action Needed to Stop Seafood Fraud in the United States. A report by Oceana. https://doi.org/10.31230/osf.io/sbm8h

631 White, D. J., Svellingen, C., \& Strachan, N. J. C. (2006). Automated measurement of species and length of fish by computer vision. Fisheries Research, 80, 203-210.

634 Wu, D. \& Sun, D. (2013). Potential of time series-hyperspectral imaging (TS-HSI) for non-invasive determination of microbial spoilage of salmon flesh. Talanta, 111,

637 Zhang, Y., Wang, X., Shan, J., Zhao, J., Zhang, W., Liu, L., \& Wu, F. (2019). Hyperspectral imaging based method for rapid detection of microplastics in the intestinal tracts of fish. Environmental Science \& Technology, 53, 5151-5158. https://doi.org/10.1021/acs.est.8b07321 
641 Zhang, Z., Chen, S., \& Liang, Y. (2010). Baseline correction using adaptive iteratively 642 reweighted penalized least squares. Analyst, 135(5), 1138-1146.

$643 \quad \underline{\text { http://doi.org/10.1039/B922045C }}$

644 Zhu, F., Zhang, D., He, Y., Liu, F., \& Sun, D. (2013). Application of visible and near

645 infrared hyperspectral imaging to differentiate between fresh and frozen-thawed

646 fish fillets. Food and Bioprocess Technology, 6(10), 2931-2937.

$647 \quad$ https://doi.org/10.1007/s11947-012-0825-6

648

649

650

651

652

653

654

655

656

657

658

659

660

661

662

663 


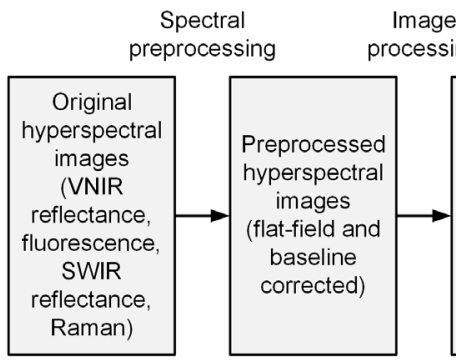

$$
\text { Spectral }
$$

Dataset generation

Fish species \& freshness labels

Two-fold

\begin{tabular}{|c|c|c|c|c|c|}
\hline & & ener & fresh & abels & dation \\
\hline \multirow{5}{*}{$\begin{array}{c}\text { Spatial mask } \\
\text { and } 10 \times 10 \\
\text { average- } \\
\text { window } \\
\text { images }\end{array}$} & \multirow{5}{*}{$\begin{array}{c}\text { Mean spectra } \\
\text { from average } \\
\text { windows } \\
\text { (VNIR } \\
\text { reflectance, } \\
\text { fluorescence, } \\
\text { SWIR } \\
\text { reflectance, } \\
\text { Raman) }\end{array}$} & \multirow{4}{*}{\multicolumn{2}{|c|}{$\begin{array}{c}\text { Full spectra } \\
\text { First ten PCA } \\
\text { components }\end{array}$}} & \multirow{5}{*}{$\begin{array}{l}\text { Machine } \\
\text { learning } \\
\text { classifications } \\
\text { for species } \\
\text { and freshness } \\
\text { using } 24 \\
\text { classifiers in } \\
\text { six categories }\end{array}$} & \multirow{5}{*}{$\begin{array}{l}\text { Accuracies of } \\
24 \text { classifiers } \\
\text { for four types } \\
\text { of spectra in } \\
\text { three datasets } \\
\text { (full, PCA, } \\
\text { and selected } \\
\text { bands) }\end{array}$} \\
\hline & & & & & \\
\hline & & & & & \\
\hline & & & & & \\
\hline & & SFS & $\begin{array}{c}\text { Spectra at } \\
\text { selected } \\
\text { bands }\end{array}$ & & \\
\hline
\end{tabular}

666 Fig. 1. Flowchart of spectral and image processing and machine learning classifications.

667

668

669

670

671

672

673

674

675

676

677

678

679

680

681

682

683 
(a)
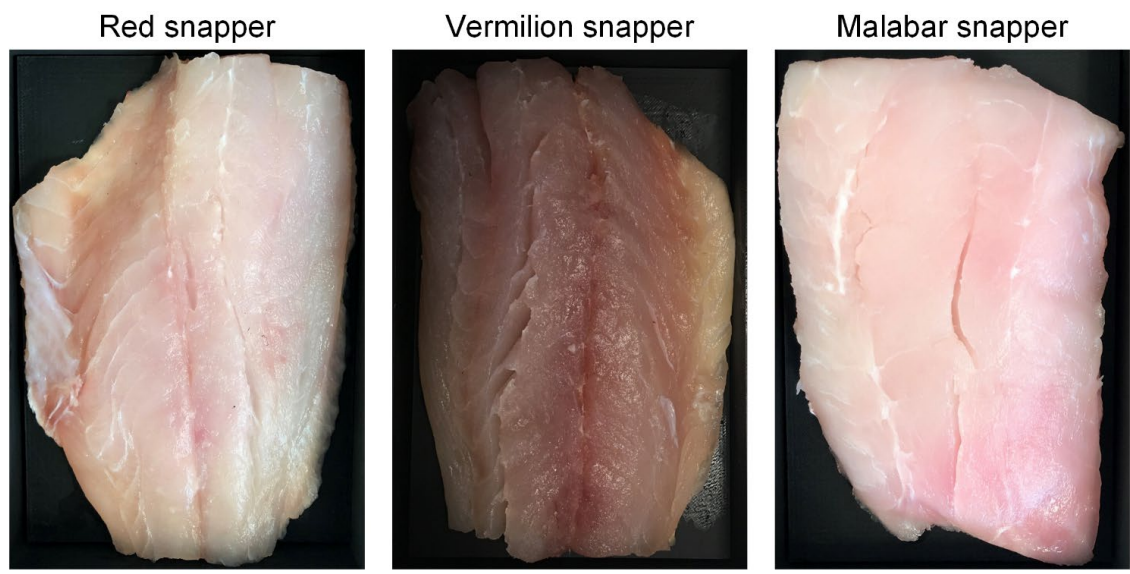

Summer flounder

White bass

Tilapia
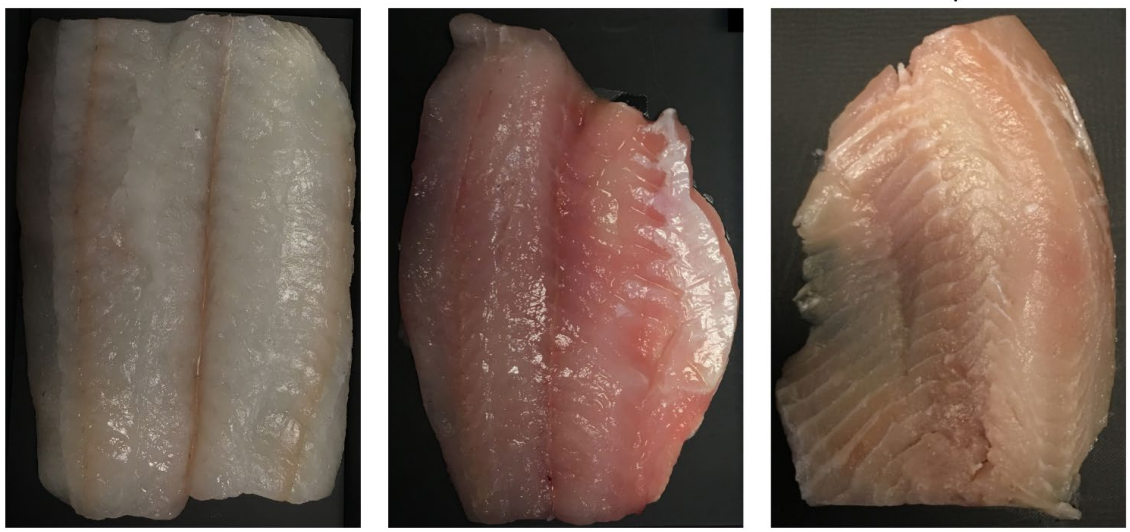

686

(b)

Red snapper as received (AR)

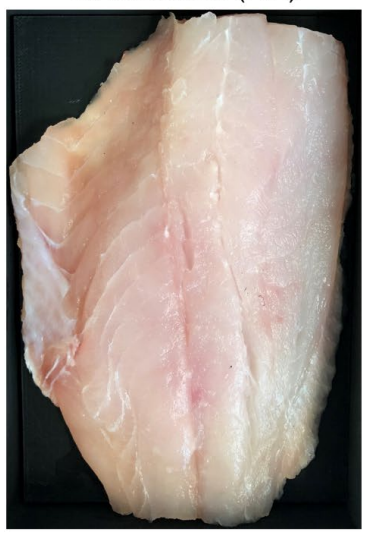

Frozen/thawed 1st cycle (FT1)

rozen/thawed 2nd cycle (FT2)
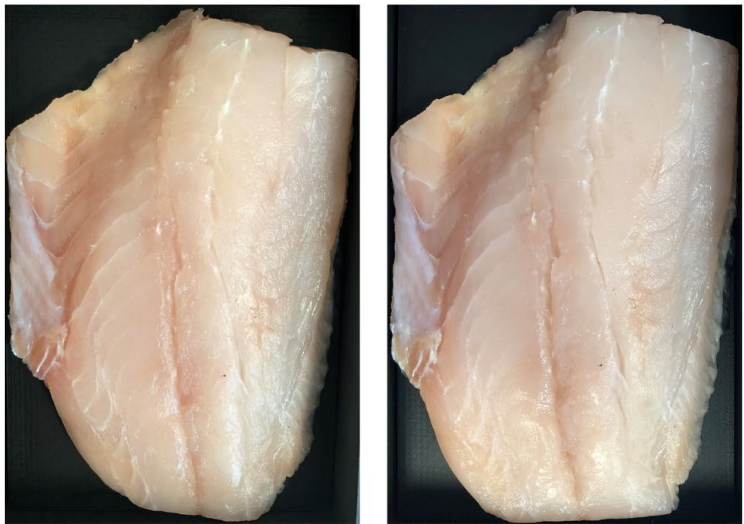

688 Fig. 2. Pictures of fish fillet samples: (a) six types of fish used for the species differentiation

689 study and (b) an example red snapper fillet used for the freshness evaluation study. 

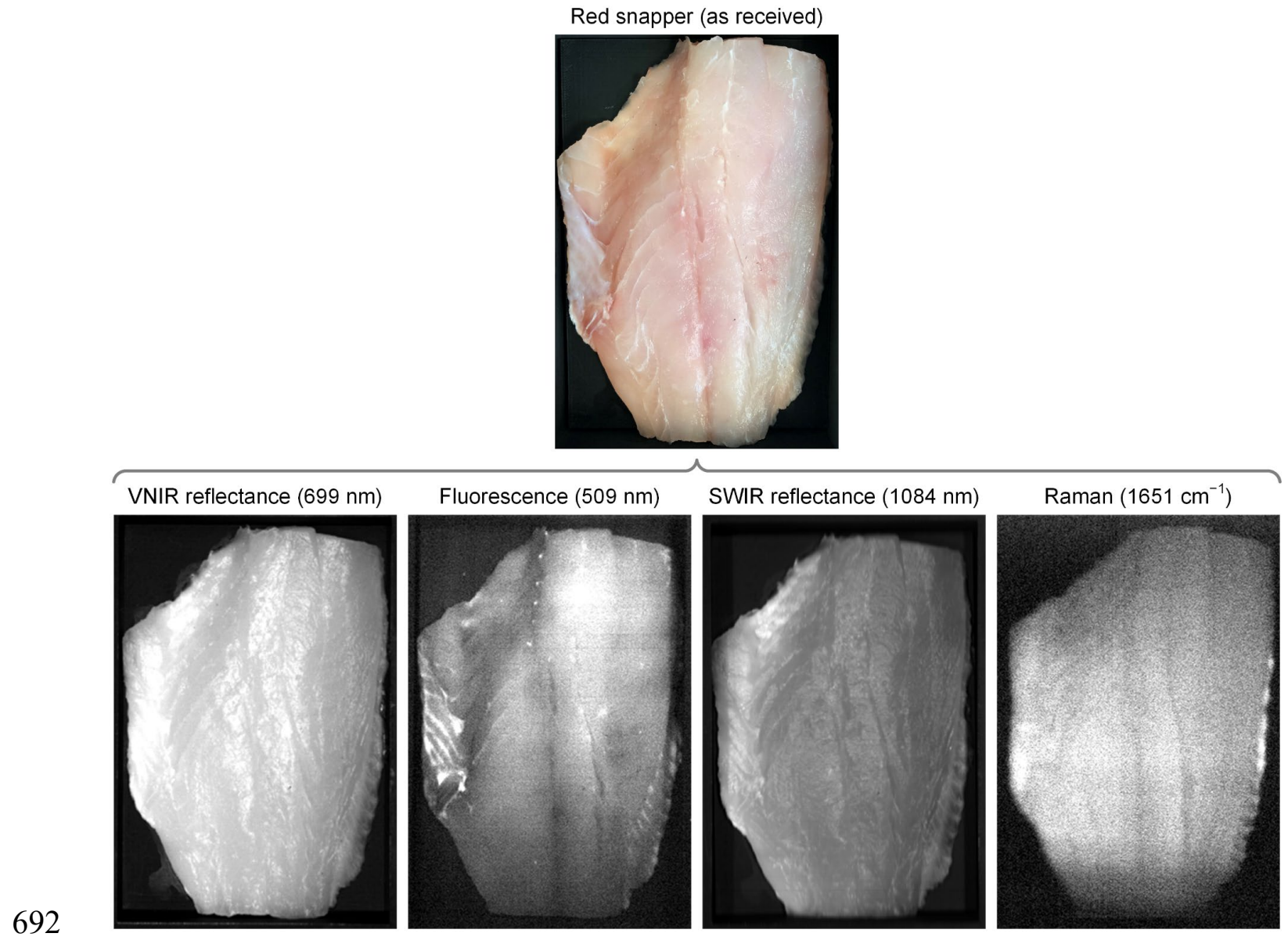

693 Fig. 3. Four single-band images extracted from hyperspectral data collected from a red

694 snapper fillet.

695

696

697

698

699

700

701

702 
(a)
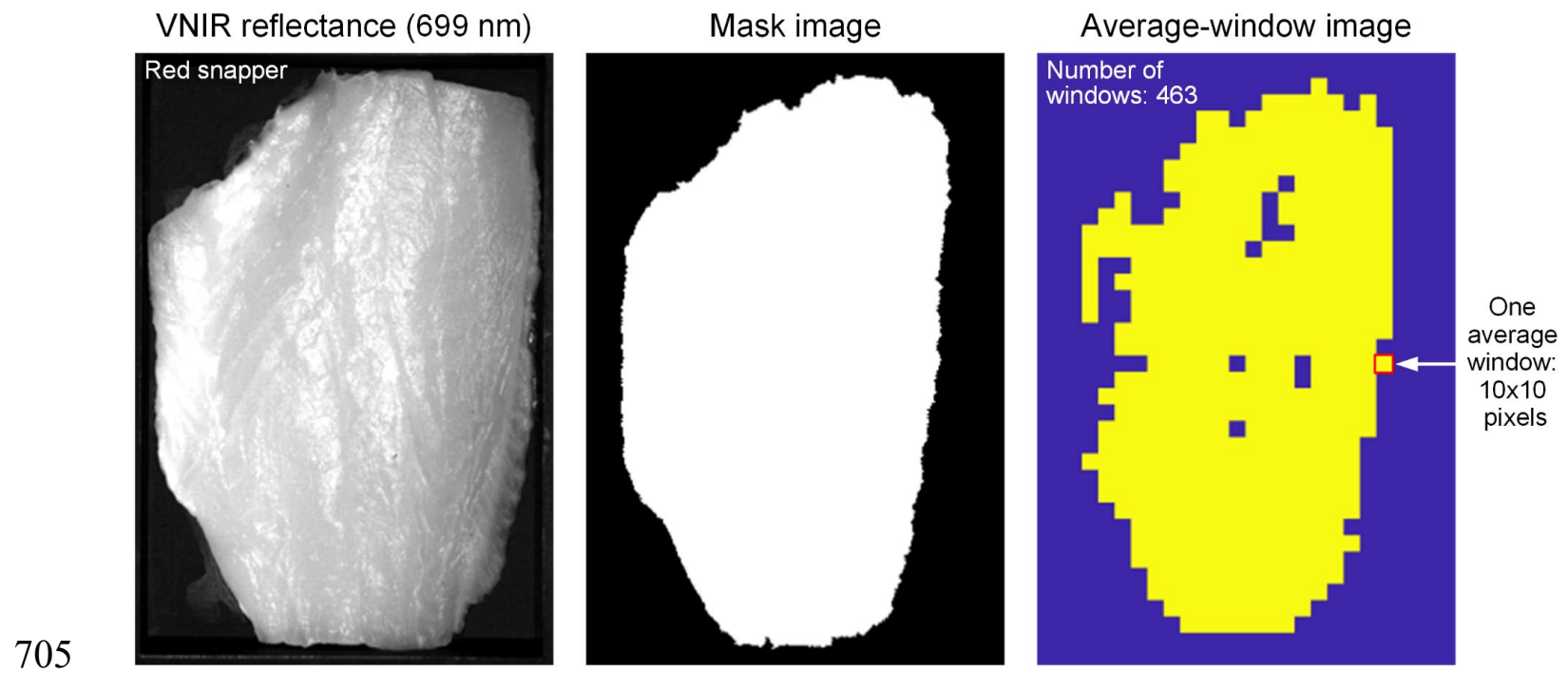

706

(b)

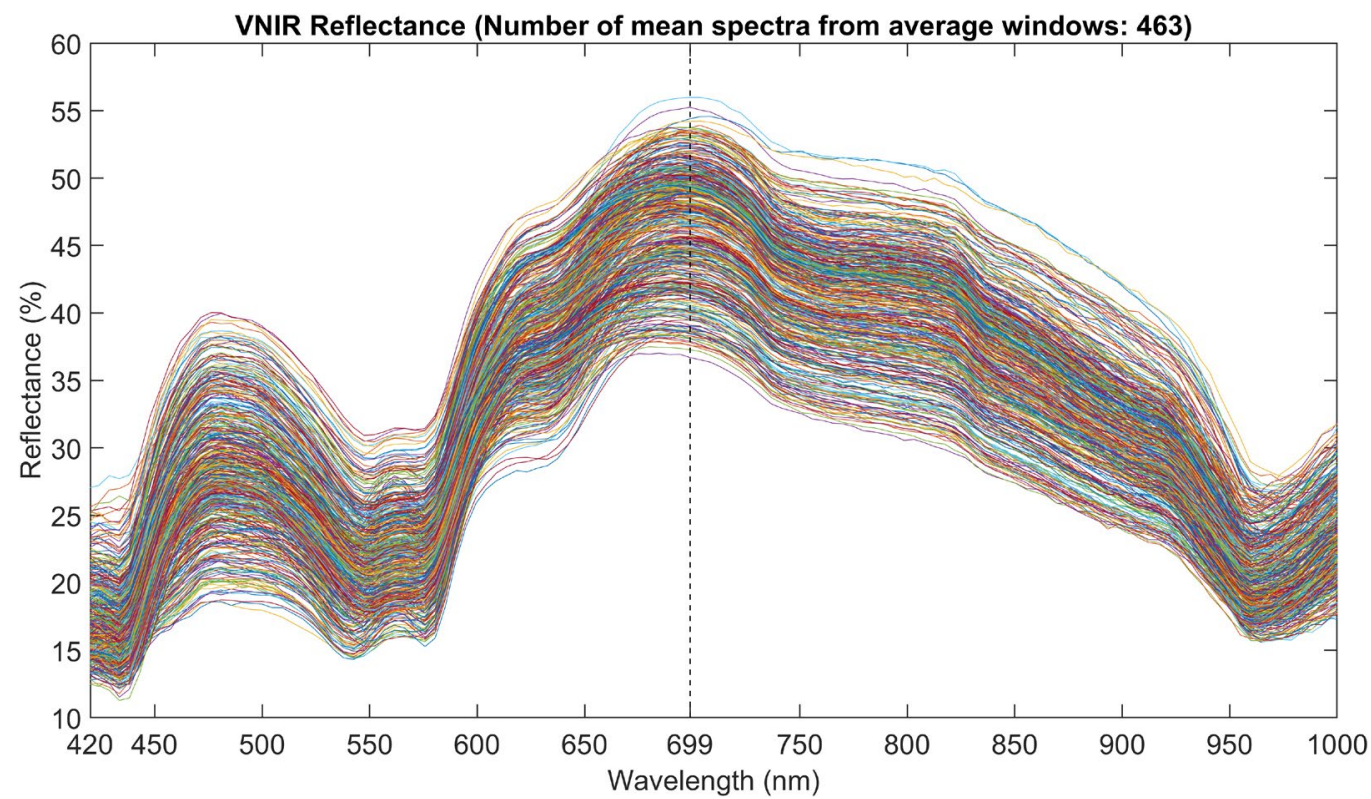

708 Fig. 4. Extraction of spectra from a VNIR hyperspectral reflectance image of a red snapper

709 fillet: (a) a mask image created using a single-band image at $699 \mathrm{~nm}$ and an average-

710 window image used to obtain mean spectra within each of the $10 \times 10$ pixel regions, and (b)

711 mean reflectance spectra from 463 average windows. 
(a)

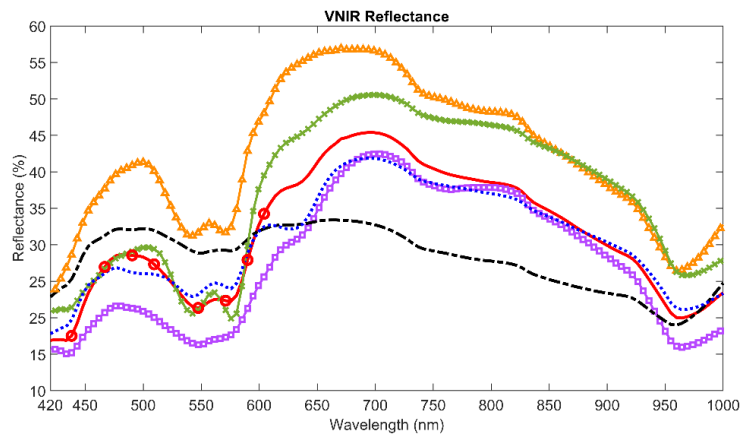

(c)

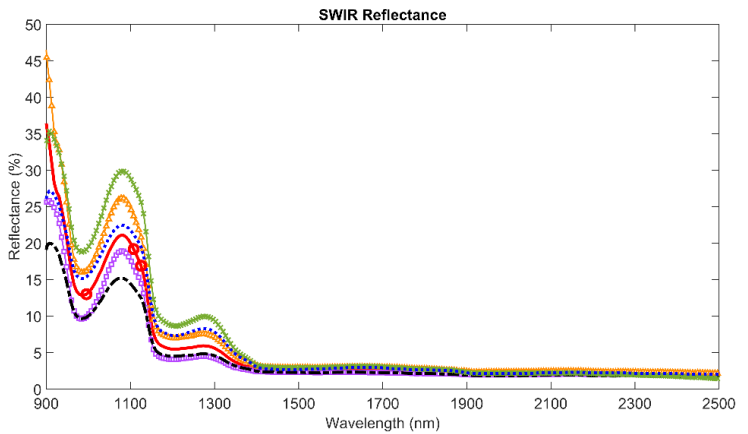

(b)

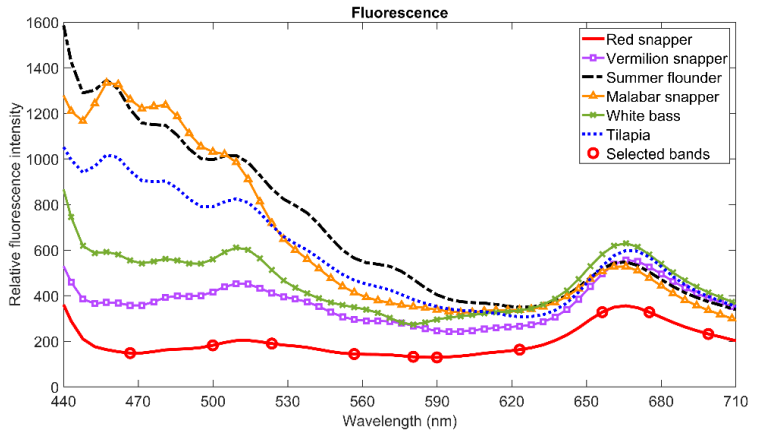

(d)

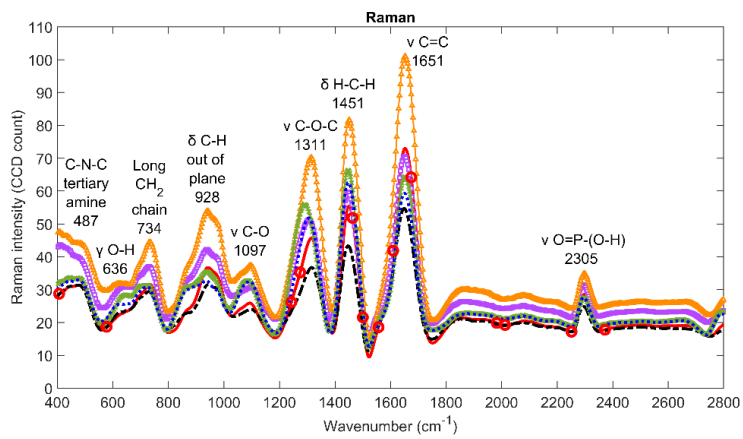

715

Fig. 5. Mean spectra of six fish species: (a) VNIR reflectance, (b) fluorescence, (c) SWIR

717 reflectance, and (d) Raman. Selected bands for fish species classifications are marked on

718 each spectrum of the red snapper sample.

719 
(a)

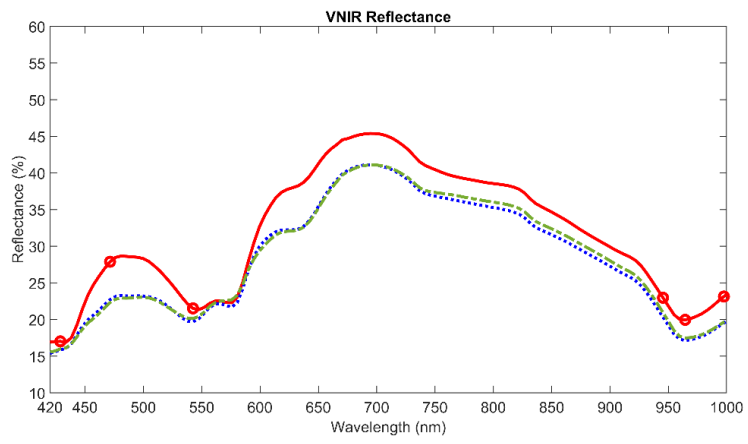

(c)

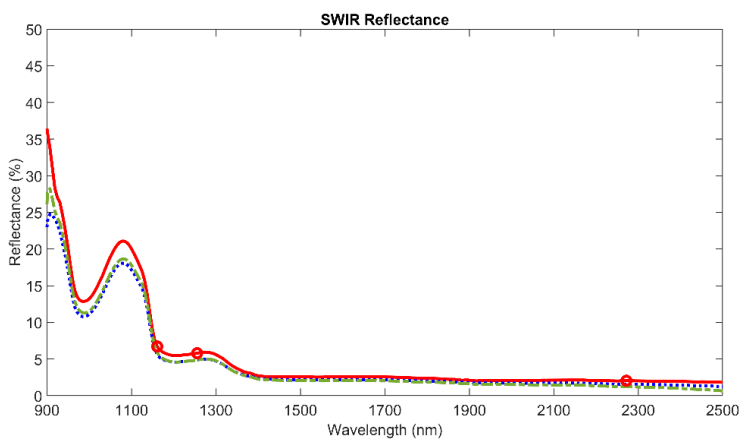

(b)

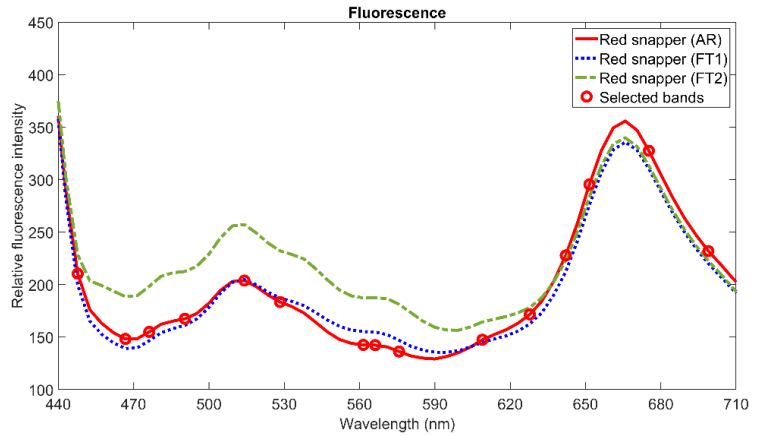

(d)

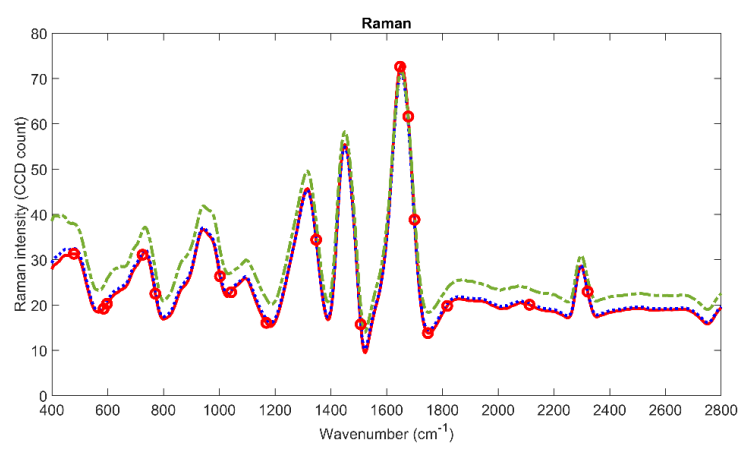

730 Fig. 6. Mean spectra of red snapper fillets as received (AR) and after two freeze-thaw

731 cycles (FT1 and FT2): (a) VNIR reflectance, (b) fluorescence, (c) SWIR reflectance, and

732 (d) Raman. Selected bands for fish freshness classifications are marked on each spectrum

733 of the AR red snapper fillet. 
(a)

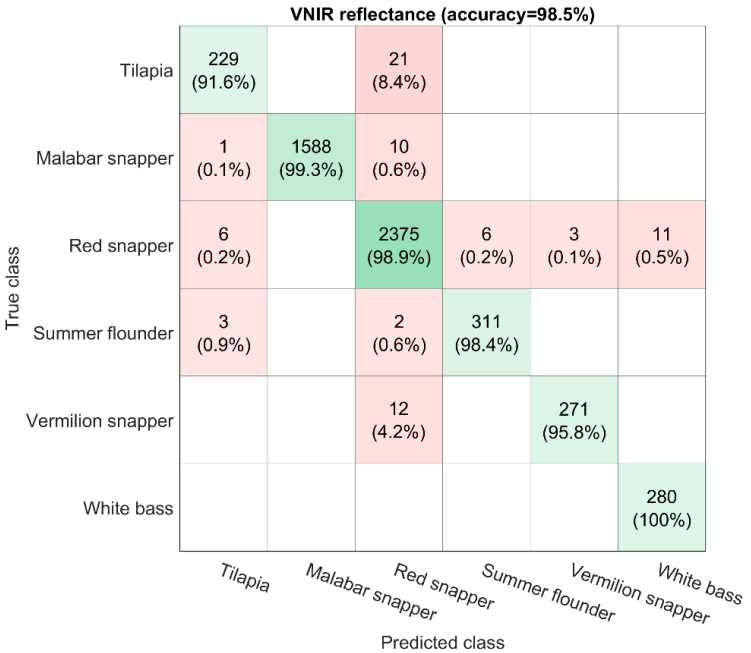

(c)

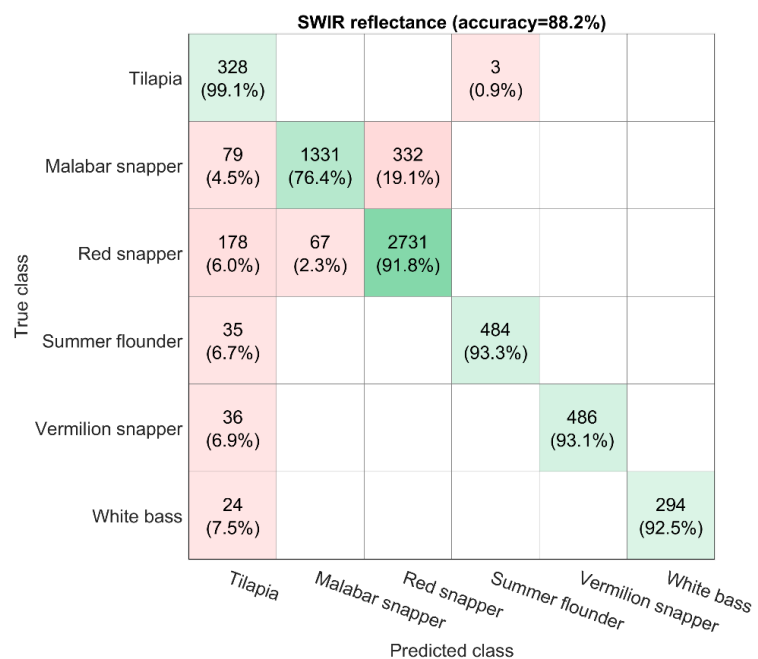

(b)

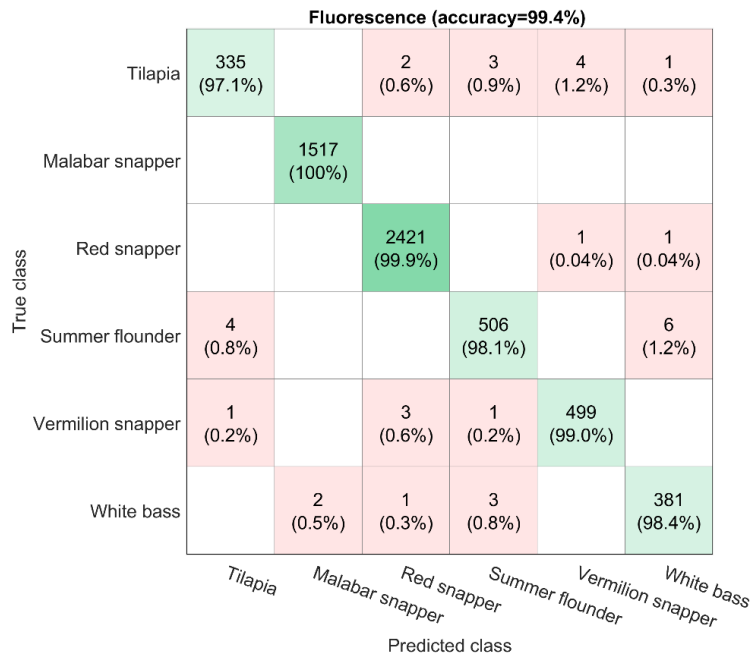

(d)

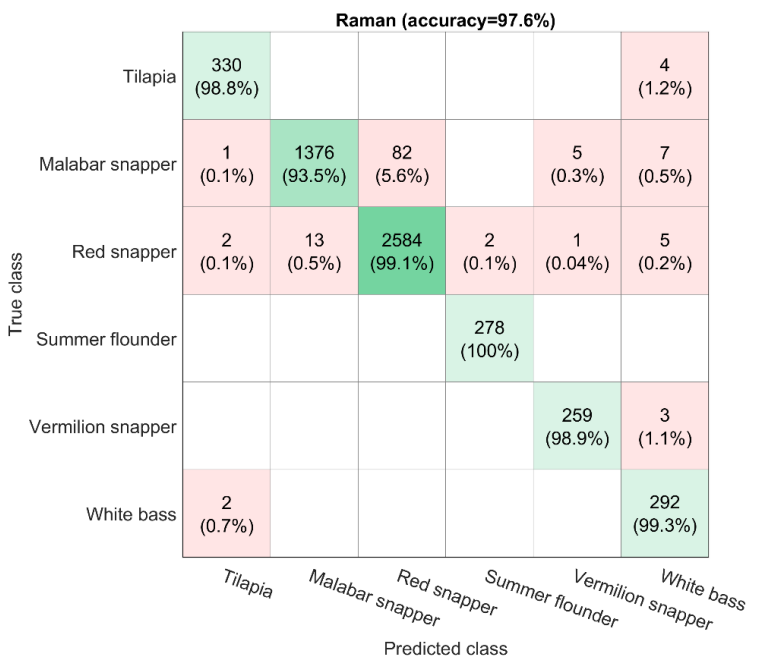

744 Fig. 7. Confusion matrices for fish species classifications using linear support vector

745 machines with full spectral data of (a) VNIR reflectance, (b) fluorescence, (c) SWIR

746 reflectance, and (d) Raman. 
(a)

752

753

754

755

756

757 Fig. 8. Species classification accuracies for fillets from six types of fish by 24 machine

758 learning classifiers using (a) full spectra, (b) first ten components of PCA, and (c) bands

759 selected by SFS.

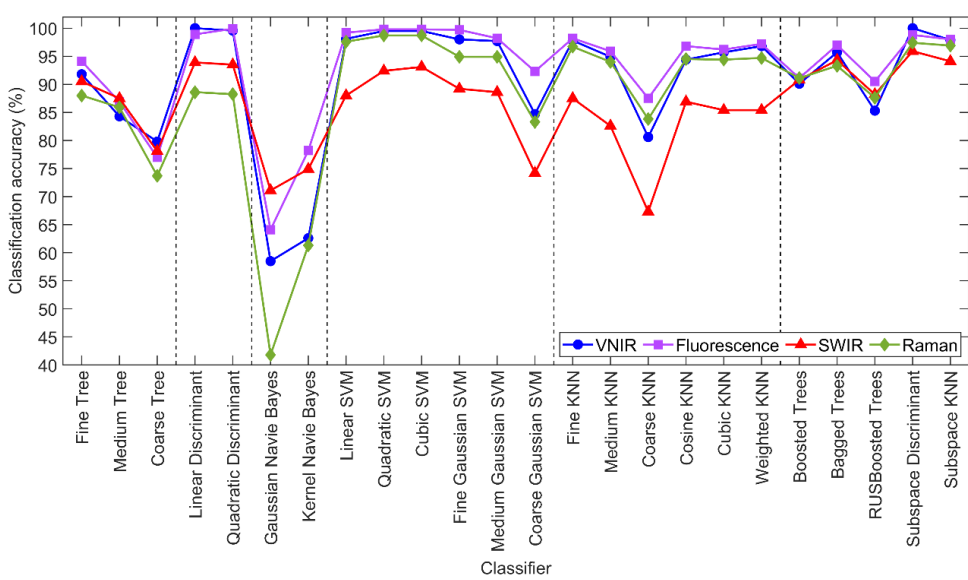

(b)

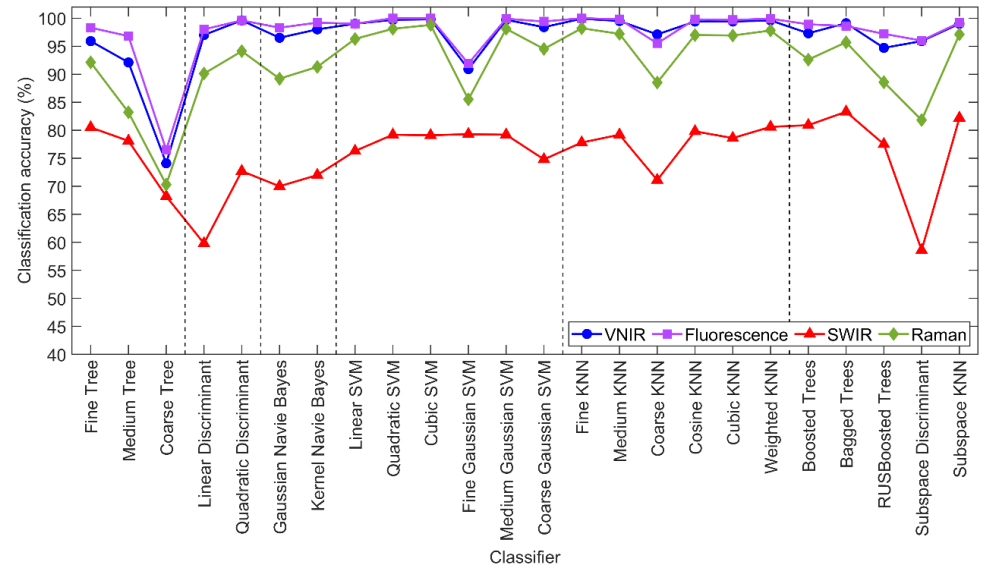

(c)

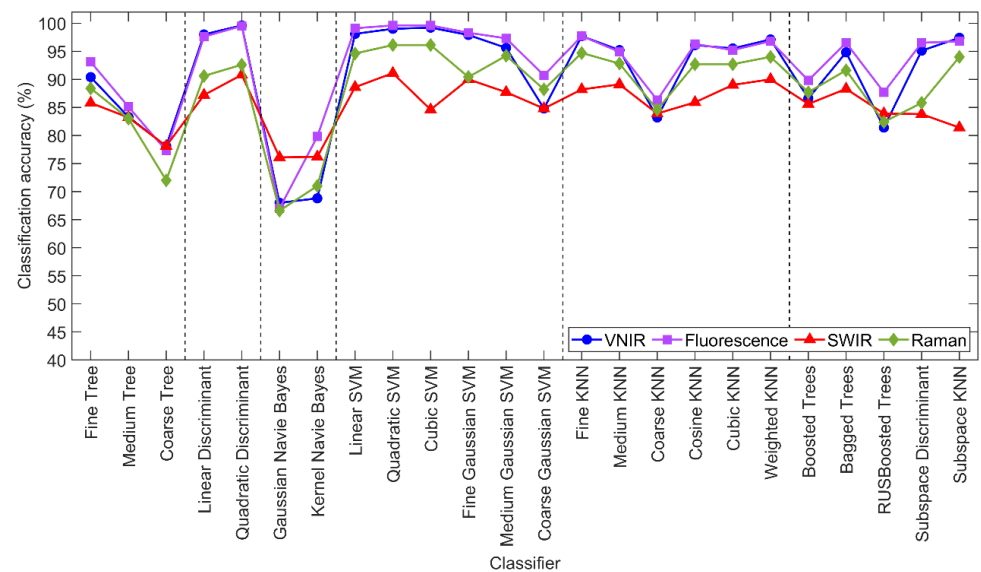


(a)

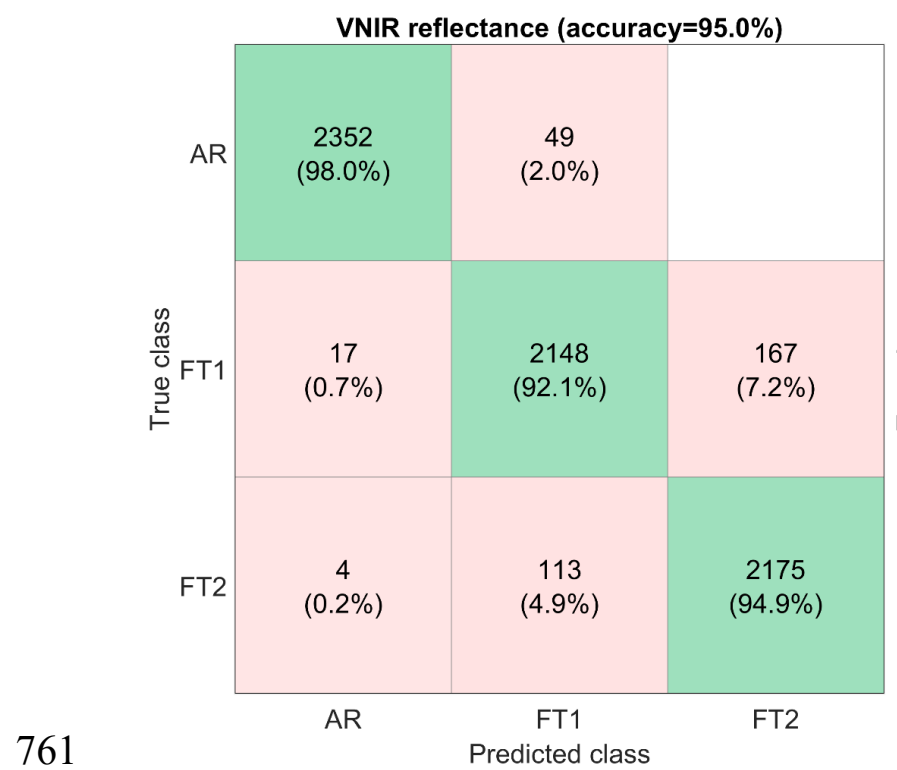

(c)

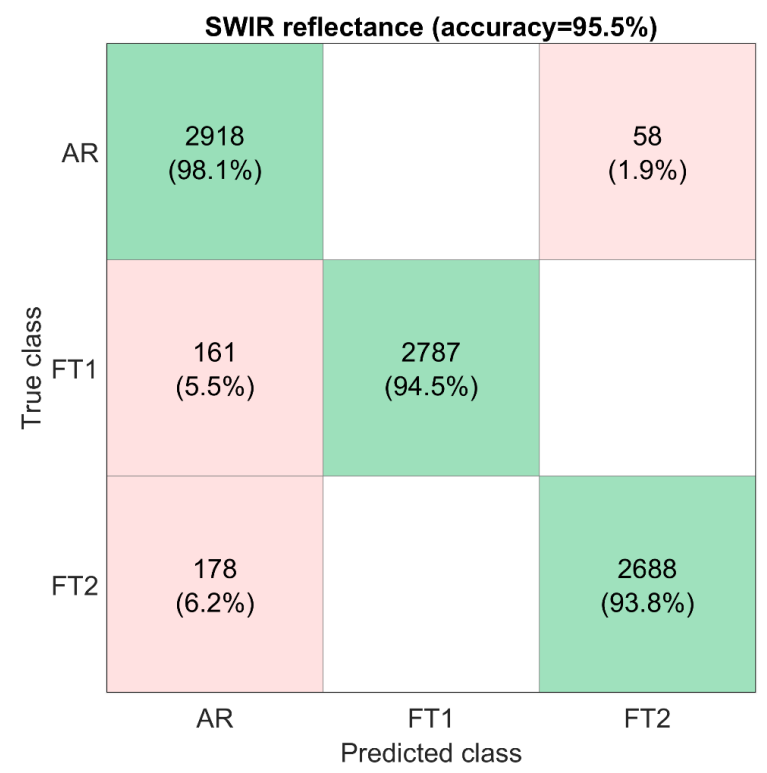

(b)

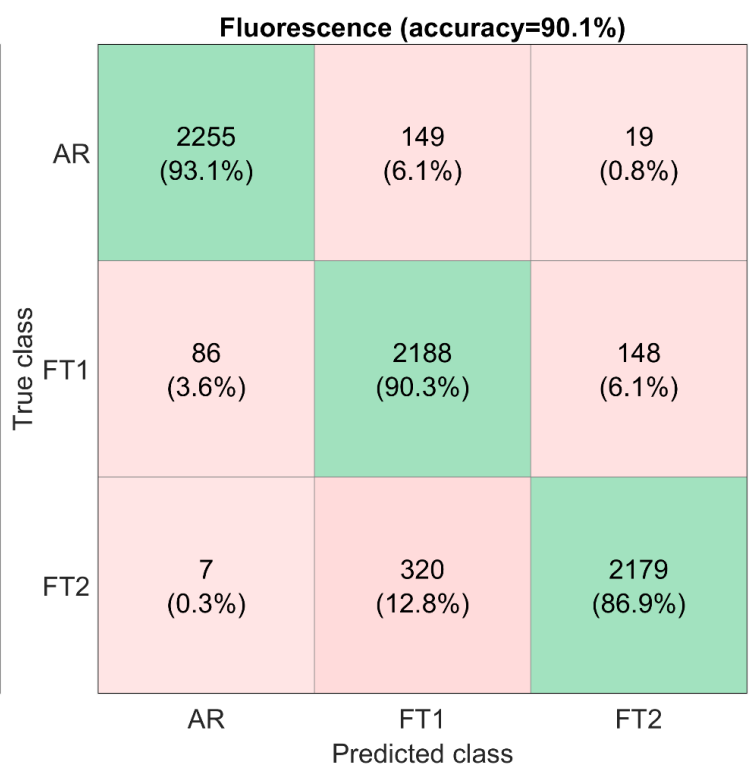

(d)

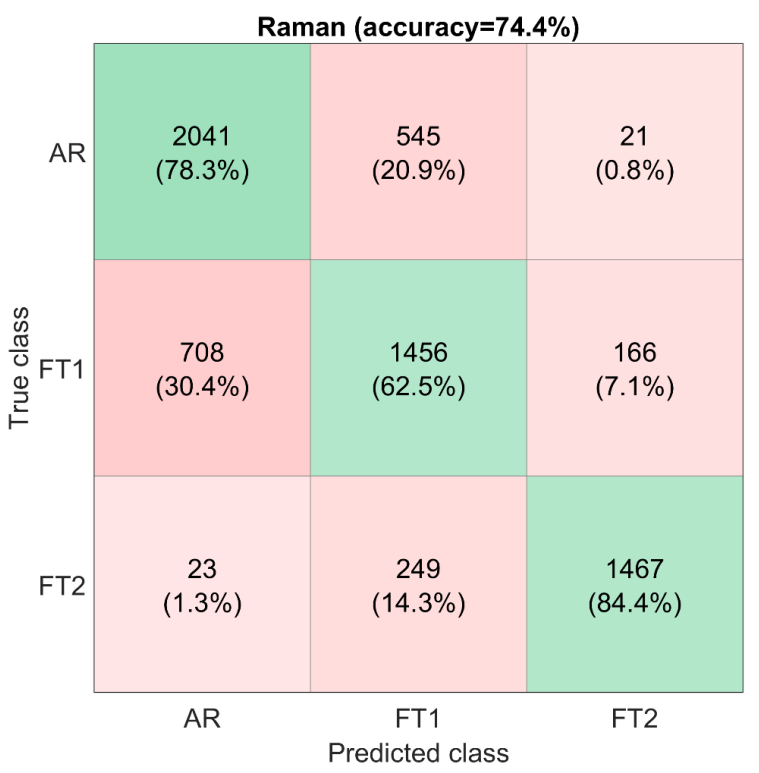

Fig. 9. Confusion matrices for freshness classifications of red snapper fillets (including as-

764 received (AR) and after two freeze-thaw cycles (FT1 and FT2)) using linear support vector

765 machines with full spectral data of (a) VNIR reflectance, (b) fluorescence, (c) SWIR

766 reflectance, and (d) Raman. 
(a)

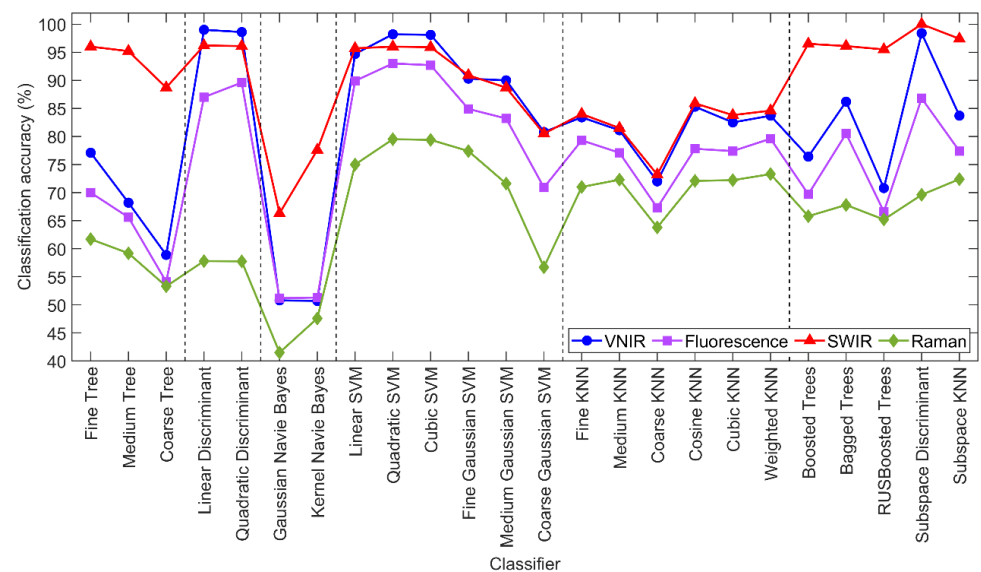

(b)

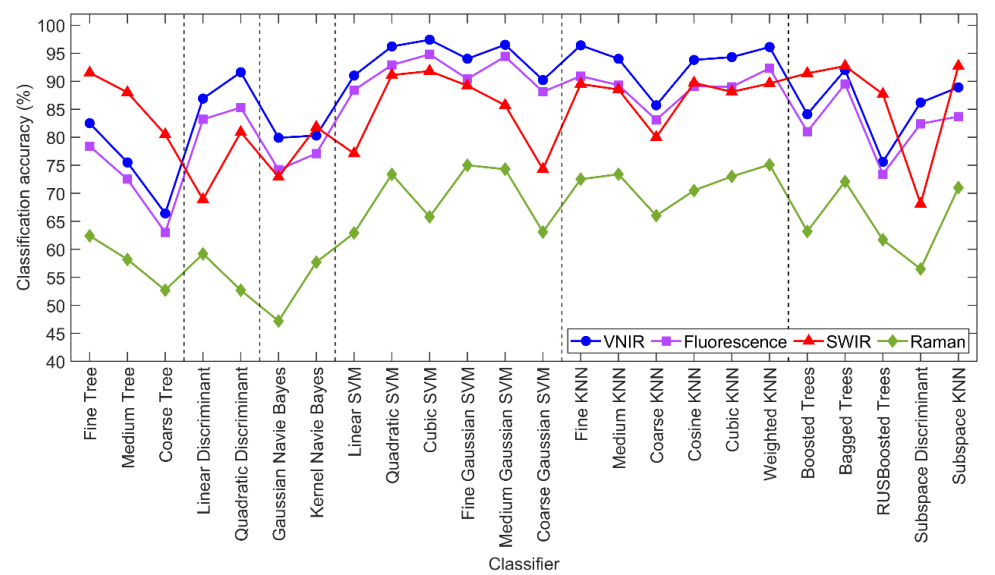

(c)

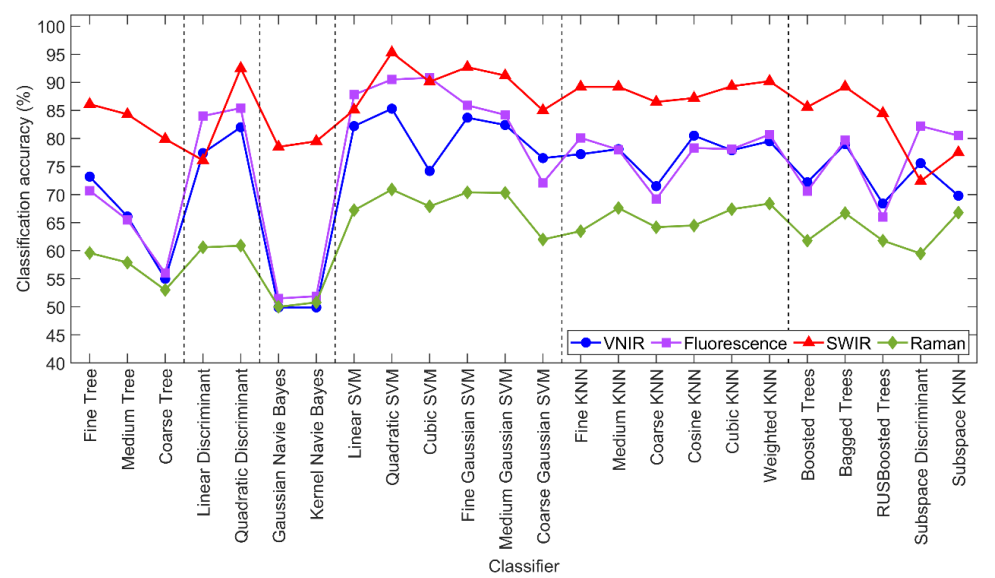

774 Fig. 10. Freshness classification accuracies for as-received and frozen-thawed red snapper

775 fillets by 24 machine learning classifiers using (a) full spectra, (b) first ten components of 776 PCA, and (c) bands selected by SFS. 
778 Table 1. Key components and settings of three line-scan hyperspectral imaging systems

779 used to collect four types of image data from fish fillets.

\begin{tabular}{|c|c|c|c|c|}
\hline $\begin{array}{l}\text { Components and } \\
\text { settings }\end{array}$ & $\begin{array}{l}\text { Reflectance } \\
\text { (VNIR) }\end{array}$ & Fluorescence & $\begin{array}{l}\text { Reflectance } \\
\text { (SWIR) }\end{array}$ & Raman \\
\hline Light source & $\begin{array}{l}\text { Quartz tungsten } \\
\text { halogen light }\end{array}$ & $365 \mathrm{~nm}$ UV LEDs & $\begin{array}{l}\text { Gold-coated } \\
\text { halogen light }\end{array}$ & $785 \mathrm{~nm}$ line laser \\
\hline $\begin{array}{l}\text { Imaging } \\
\text { spectrograph }\end{array}$ & $\begin{array}{l}\text { Hyperspec-VNIR } \\
\text { (Headwall) }\end{array}$ & $\begin{array}{l}\text { Hyperspec-VNIR } \\
\text { (Headwall) }\end{array}$ & $\begin{array}{l}\text { Hyperspec-SWIR } \\
\text { (Headwall) }\end{array}$ & $\begin{array}{l}\text { ImSpector R10E } \\
\text { (Specim) }\end{array}$ \\
\hline Detector & $\begin{array}{l}\text { 14-bit EMCCD } \\
\text { camera }\end{array}$ & $\begin{array}{l}\text { 14-bit EMCCD } \\
\text { camera }\end{array}$ & $\begin{array}{l}\text { 16-bit MCT array } \\
\text { detector }\end{array}$ & $\begin{array}{l}\text { 16-bit CCD } \\
\text { camera }\end{array}$ \\
\hline $\begin{array}{l}\text { Focal length of } \\
\text { lens }\end{array}$ & $23 \mathrm{~mm}$ & $23 \mathrm{~mm}$ & $25 \mathrm{~mm}$ & $23 \mathrm{~mm}$ \\
\hline Spectral range & $419-1007 \mathrm{~nm}$ & $438-718 \mathrm{~nm}$ & $842-2532 \mathrm{~nm}$ & $103-2831 \mathrm{~cm}^{-1}$ \\
\hline $\begin{array}{l}\text { Spatial resolution } \\
\text { along IFOV }\end{array}$ & $0.4 \mathrm{~mm} /$ pixel & $0.4 \mathrm{~mm} /$ pixel & $0.4 \mathrm{~mm} /$ pixel & $0.4 \mathrm{~mm} /$ pixel \\
\hline $\begin{array}{l}\text { Line-scan } \\
\text { incremental size }\end{array}$ & $0.4 \mathrm{~mm}$ & $0.4 \mathrm{~mm}$ & $0.4 \mathrm{~mm}$ & $0.4 \mathrm{~mm}$ \\
\hline Scan number & 280 & 280 & 350 & 260 \\
\hline Exposure time & $0.015 \mathrm{~s}$ & $0.3 \mathrm{~s}$ & $0.006 \mathrm{~s}$ & $4.0 \mathrm{~s}$ \\
\hline Scan time & $1 \mathrm{~m} 20 \mathrm{~s}$ & $2 \mathrm{~m} 24 \mathrm{~s}$ & $15 \mathrm{~s}$ & $20 \mathrm{~m} 20 \mathrm{~s}$ \\
\hline Hypercube size & $500 \times 280 \times 125$ & $500 \times 280 \times 60$ & $384 \times 350 \times 287$ & $400 \times 260 \times 846$ \\
\hline
\end{tabular}


786 Table 2. Numbers of fish fillet samples and mean spectra extracted from hyperspectral

787 images used for species classifications.

\begin{tabular}{lccccc}
\hline \multicolumn{1}{c}{ Fish species } & Fillet number & $\begin{array}{c}\text { Reflectance } \\
\text { (VNIR) }\end{array}$ & Fluorescence & $\begin{array}{c}\text { Reflectance } \\
\text { (SWIR) }\end{array}$ & Raman \\
\hline Red snapper & 6 & 2401 & 2423 & 2976 & 2607 \\
Vermilion snapper & 1 & 283 & 504 & 522 & 262 \\
Malabar snapper & 4 & 1599 & 1517 & 1742 & 1471 \\
Summer flounder & 1 & 316 & 516 & 519 & 278 \\
White bass & 1 & 280 & 387 & 318 & 294 \\
Tilapia & 1 & 250 & 345 & 331 & 334 \\
\hline Total & 14 & 5129 & 5692 & 6408 & 5246 \\
\hline
\end{tabular}

788 
799 Table 3. Numbers of red snapper fillet samples and mean spectra extracted from

800 hyperspectral images used for freshness classifications.

\begin{tabular}{cccccc}
\hline Red snapper & Fillet number & $\begin{array}{c}\text { Reflectance } \\
\text { (VNIR) }\end{array}$ & Fluorescence & $\begin{array}{c}\text { Reflectance } \\
\text { (SWIR) }\end{array}$ & Raman \\
\hline As received (AR) & 6 & 2401 & 2423 & 2976 & 2607 \\
$\begin{array}{c}\text { After 1st freeze- } \\
\text { thaw cycle (FT1) }\end{array}$ & 6 & 2332 & 2422 & 2948 & 2330 \\
$\begin{array}{c}\text { After 2nd freeze- } \\
\text { thaw cycle (FT2) }\end{array}$ & 6 & 2292 & 2506 & 2867 & 1739 \\
\hline Total & 6 & 7025 & 7351 & 8791 & 6676 \\
\hline
\end{tabular}

801 